\title{
Vortex Containment for the Gaseous-Fission Rocket $^{\dagger}$
}

\author{
JACK L. KERREBROCK* AND ROBERT V. MEGHREBLIAN** \\ California Institute of Technology
}

\begin{abstract}
Summary
The nuclear rocket is potentially capable of much higher specific impulse than any chemically fueled rocket, because of the high energy content per unit mass of the fissionable material. While a part of this potential can be realized by use of a low molecular weight propellant heated by solid fuel plates, it seems clear that the full potential can be realized only if the fissionable material can be retained in gaseous form, and its fission energy transferred directly to the propellant.

The purpose of this paper is to consider the possibility of retaining the fissionable material, in gaseous form, in the pressure field of a vortex, while the low molecular weight propellant diffuses radially inward through it. A two-dimensional, laminar, vortex flow is studied, the propellant being introduced tangentially at the periphery and withdrawn at the center.

The mass-flow capacity of the vortex, per unit of vortex length, is found to be independent of the vortex diameter, so that a large number of small-diameter vortices filling a given volume will have a much larger mass-flow capacity than a single vortex of the same volume. At tangential Mach numbers of the order of unity in a mixture of hydrogen and plutonium, the mass-flow capacity is of the order of 0.01 pounds per second-foot.

If the vortex pressure gradient decreases monotonically outward, it is possible to maintain the heavy gas in a highly peaked distribution, away from both the center and the periphery of the vortex; however, for very high heating rates, or very large temperature ratios, the pressure gradient cannot decrease monotonically outward, and in this case the concentration of heavy gas near the periphery of the vortex will be high.

Insofar as the vortex-containment mechanism limits the gaseous nuclear rocket, its performance potential is very high. It may be that its performance will actually be limited by other factors, such as radiant heat transfer from the gas to solids, the difficulty of maintaining fissionable material in gaseous form, and the difficulty of generating vortices with the required low radial mass-flow rates and high tangential velocities. The potential of the gaseous fission rocket seems to warrant intensive study of these problems.
\end{abstract}

\section{Introduction}

T HE NUClEAR ROCKET, in which the fission reaction acts as a source of energy to heat the propellant, which is then expanded through a nozzle to produce thrust, is potentially capable of much higher specific impulse than is any chemically fueled rocket. This is because the energy content of the heated propellant per unit mass is not limited, as in the case of the chemical rocket, by the chemical energy content of the pro-

Received May 20, 1960.

$\dagger$ This paper is based largely upon the analysis and results of reference 1 , which was declassified December 21, 1959.

* Senior Research Fellow, Daniel and Florence Guggenheim Jet Propulsion Center.

** Chief of the Physical Sciences Division, Jet Propulsion Laboratory. pellants. The energy content of the fissionable material per unit mass is in fact so high that it need not be used as propellant; its energy may be extracted and used to heat a separate propellant.

However, the problem of transferring the energy from the fissioning fuel to the propellant makes full realization of this great potential difficult. A portion of it can be realized by choosing a propellant with a very low molecular weight, so that even with the propellant temperature limited to the values obtainable with heat transfer from fissionable materials in solid form, the propellant enthalpy, and hence the specific impulse, is considerably above that attainable with chemical propellants. It seems likely that, with hydrogen as the propellant, values of specific impulse ranging from 800 seconds to perhaps 1,200 seconds may be obtained, depending on the pressure at which the hydrogen is heated.

It seems clear, though, that the full potential of the nuclear rocket can be realized only if the fissionable material is retained in gaseous form, and heat is transferred from it to the propellant without the interposition of any solid surface. Two methods are available for this energy transfer: radiation and direct molecular collisions. Of these, we shall consider only the latter. To effect the direct transfer of the kinetic energy of the fission fragments to the propellant by molecular collisions, the fissionable material and propellant must be in intimate gaseous mixture within a nuclear reactor in which a chain reaction can be supported. It is readily demonstrated that the requirement for nuclear criticality, together with the desire for large amounts of heat addition to the gaseous propellant, dictate rather high concentrations of fissionable material in the gas mixture. If this mixture is then simply exhausted through a nozzle, the loss of fissionable material is prohibitive from the standpoint of cost. Thus, it is essential that the fissionable material be retained within the reactor, while the propellant flows through it.

The purpose of this paper is to consider the possibility of containing the fissionable material within a vortex flow. The strong radial pressure gradients which can be generated in vortices are capable of producing appreciable diffusion of a light propellant gas, such as hydrogen, through a heavy gas, such as plutonium. Thus, it seems possible that a distribution of fissionable gas may be held in a vortex while the lighter propellant gas diffuses radially inward through it, absorbing the fission energy. One possible imple- 
mentation of this concept is shown schematically in Fig. 1. The propellant gas, and enough fissionable gas to make up losses, would be introduced with a high tangential velocity at the periphery of the vortex tube, through a series of jets distributed over its surface. The propellant would then diffuse radially inward, through the distribution of fissionable gas, and leave the tube axially near its center.

For the purposes of analysis, this flow will be idealized to a two-dimensional vortex flow, into which the fluid is introduced uniformly at the periphery with a tangential velocity, and from which it is withdrawn at some radius near the center, as by a cylindrical sink at that radius. No attempt will be made to treat the threedimensional flow near the axis; it is felt that this flow is not of crucial importance to the containment mechanism. A question does arise as to the proper boundary conditions to be applied at the inner and outer radii of the two-dimensional flow; this question will be discussed in some detail.

The flow will be assumed to be laminar. It may seem that this assumption is rather extreme, because the Reynolds number of the flow, based on the high tangential velocity and the tube diameter, must be large, and the resulting turbulent mixing would tend to invalidate the laminar calculation. However, the nature of turbulent vortex flows is not well understood, particularly when the fluid is compressible, so that conclusions based on turbulent mixing processes for radically different flows (e.g., pipe flow) may be completely erroneous. The point of view which is taken here is that the validity of the laminar diffusion calculation can best be judged by direct comparison with experiment.

Fission-fragment heating of the propellant is assumed to occur locally at the point of fission-i.e., the range of the fission fragments is assumed small compared to the radius of the vortex. At one hundred atmospheres pressure and $2,000^{\circ} \mathrm{K}$., the range of fission fragments in hydrogen is about $0.7 \mathrm{~cm} .,^{2}$ however, half of their energy is deposited in the first $0.2 \mathrm{~cm}$., hence, at this pressure and temperature, the vortex radius must be one centimeter or larger. An additional assumption, that the heating rate is proportional to the concentration of fissionable material, implies that the neutron flux within the vortex is constant. It will be assumed that the mole fraction of fissionable gas in the mixture is small. This assumption simplifies the analysis considerably, and because of the much larger mass of the fissionable atoms compared to that of the propellant atoms, is valid even when the density of fissionable material is considerably greater than that of the propellant.

Finally, the vortex flow will be assumed inviscid, so that the tangential velocity is inversely proportional to the radius. The justification for this is that the radial-inflow Reynolds number is sufficiently large for the interesting cases that viscous effects are in fact small, except near the solid outer boundaries of the vortex. Boundary-layer-like flows with high rates of shear will exist on such surfaces, and the resultant torque may make the attainment of vortices with sufficiently high tangential velocities difficult; however, this problem will not be considered here.

In the present paper, we shall be concerned with the process of diffusion of the propellant through the heavy gas, with heat addition. No detailed consideration will be given to the many other problems, such as radiant heat transfer and nuclear criticality; which must clearly be of great importance for a gaseous reactor.

Nevertheless, some estimates of the performance of gaseous nuclear rockets, as limited by the vortex containment, will be given. It must be recognized that these estimates cannot reflect the ultimate potential of the gaseous nuclear rocket with vortex containment. They can only serve to indicate the limits imposed by the containment scheme itself.

\section{Formulation of the Diffusion Problem}

For a complete description of the two-component gas mixture, two new variables must be added to the usual fluid dynamic set. These may be taken as the

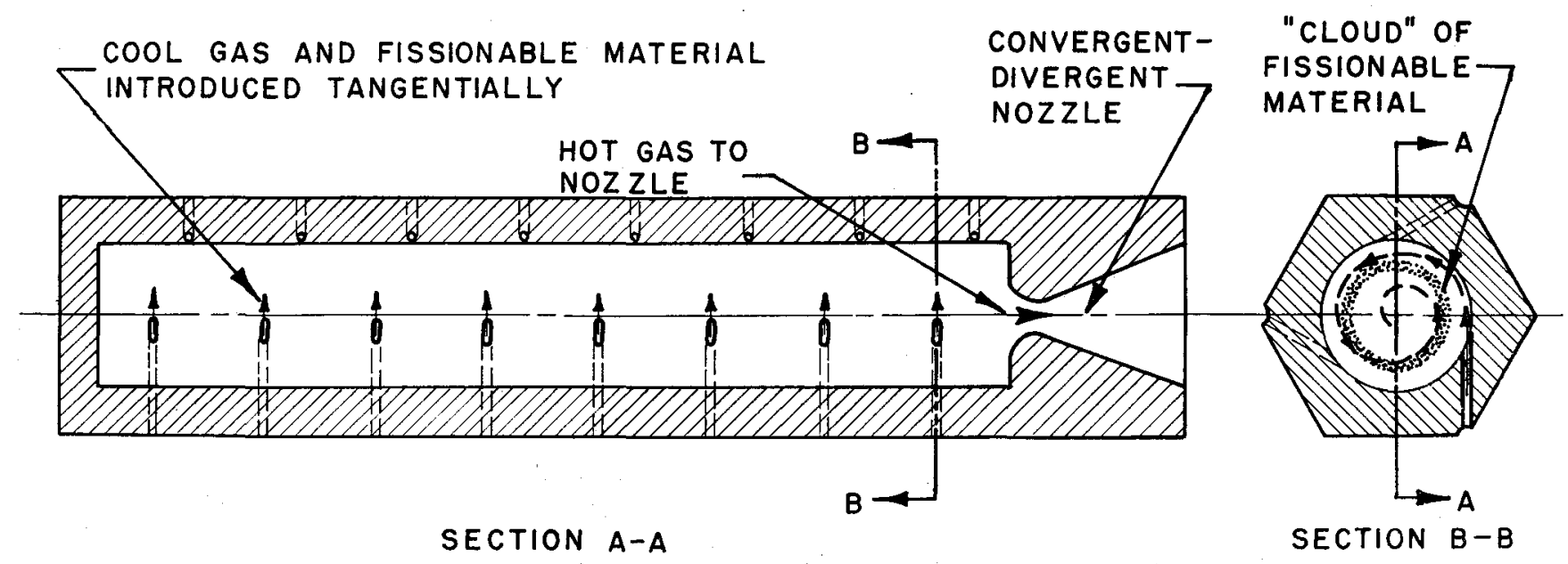

Fig. 1. Specialization of the vortex tube for rocket propulsion. 
density and velocity of one species, say the heavy gas, in which case the continuity equation for the heavy gas and the relation connecting its diffusion velocity to the pressure and concentration gradients complete the set of governing equations.

In the two-dimensional vortex flow, the continuity equation for the mixture is quite simple. If $u$ is the radial gas velocity, $\rho$ the density, and $r$ the radius, then

$$
\rho u r=\left(\mathfrak{M}_{1}+\mathfrak{M}_{2}\right) / 2 \pi
$$

where $\mathfrak{T}_{1}$ and $\mathfrak{N}_{2}$ are the constant mass flows of the light and heavy gases, respectively, per unit of vortex length. Similarly, since the two gases do not react chemically, the heavy gas satisfies the continuity equation

$$
\rho_{2}\left(u+u_{2}\right) r=\mathfrak{M}_{2} / 2 \pi
$$

where $u_{2}$ is the diffusion velocity of the heavy gas.

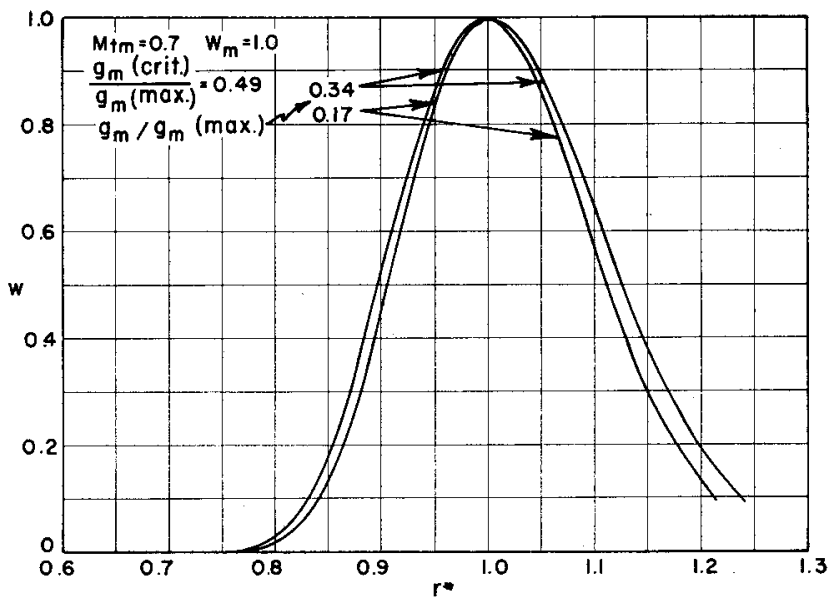

FIG. 2. Variation of density ratio, $x$, with dimensionless radius, $r^{*}$, for $g_{m}$ less than $g_{m}$ (crit).

\section{The Diffusion Equation}

Neglecting thermal diffusion, the diffusion velocity is related to the concentration and pressure gradients by $:^{3}$

$$
\begin{array}{r}
u_{2}\left(\frac{\rho}{\rho-\rho_{2}}\right)=-D_{12}\left\{\frac{n}{n-n_{2}} \frac{d \log \left(n_{2} / n\right)}{d r}+\right. \\
\left.\frac{n\left(m_{1}-m_{2}\right)}{\rho} \frac{d \log p}{d r}\right\}
\end{array}
$$

where $n$ is the total gas concentration, $p$ the total gas pressure, $m_{1}$ and $m_{2}$ the respective molar masses, and $D_{12}$ is the binary diffusion coefficient. Now by using Eq. (2) to eliminate $u_{2}$, Eq. (3) may be put in the form

$$
\begin{aligned}
& \left(\frac{\mathfrak{M}_{2}}{\mathfrak{M}_{1}+\mathfrak{M}_{2}}-\frac{\rho_{2}}{\rho}\right)=\frac{-2 \pi r \rho_{1} D_{12}}{\mathfrak{M}_{1}+\mathfrak{M}_{2}}\left(\frac{\rho_{2}}{\rho}\right) \times \\
& \left\{\frac{n}{n-n_{2}} \frac{d \log \left(n_{2} / n\right)}{d r}+\frac{n\left(m_{1}-m_{2}\right)}{\rho} \frac{d \log p}{d r}\right\}
\end{aligned}
$$

It is convenient to rewrite this expression in terms of the ratio of fissionable material and propellant densities, $w=\rho_{2} / \rho_{1}$. As was mentioned previously, it will be assumed that $n_{2} / n \ll 1$, however, because $m_{2} \gg$ $m_{1}$, it does not follow that $w \ll 1$. In terms of $w$, the diffusion equation then becomes

$$
\begin{array}{r}
\left(\frac{\mathfrak{M}_{2}}{\mathfrak{M}_{1}+\mathfrak{M}_{2}}-\frac{w}{1+w}\right)=\frac{-2 \pi r \rho_{1} D_{12}}{\mathfrak{M}_{1}+\mathfrak{M}_{2}}\left(\frac{w}{1+w}\right) \times \\
\left\{\frac{d \log w}{d r}-\frac{\left(m_{2} / m_{1}-1\right)}{1+w} \frac{d \log p}{d r}\right\}
\end{array}
$$

It will become clear later that $\mathfrak{N}_{2} / \mathfrak{M T}_{1}$ is determined by the value of $w$ at the boundary where the flow leaves the vortex (in this case, the inner boundary). Thus, $\mathfrak{M}_{2} / \mathfrak{M}$, is a characteristic value of the diffusion equation. The magnitude of this characteristic value influences the behavior of $w$ where it is of the order of $\mathfrak{N}_{2} / \mathfrak{N}_{1}$; where $w \gg \mathfrak{N}_{2} / \mathfrak{T}_{1}$, the behavior is controlled by the factor $2 \pi r p_{1} D_{12} /\left(\mathscr{T}_{1}+\mathfrak{M}_{2}\right)$, and by the behavior of the pressure gradient.

The radial flow velocity must be of the order of the diffusion velocity, and it is readily verified that it is therefore small compared to the tangential velocity. In this case, the radial momentum equation becomes simply

$$
d p / d r=\rho v^{2} / r
$$

where $v$ is the tangential velocity; using the fact that $n_{2} \ll n_{1}$, this may be written:

$$
(d \log p) /(d \log r)=(1+w) v^{2} / R T
$$

where $R$ is the gas constant for the light gas. Substituting this result into Eq. (5) and multiplying through by $(1+w) / w$ yields

$$
\begin{aligned}
& 1-\left(\frac{1+w}{w}\right)\left(\frac{\mathfrak{N}_{2}}{\mathfrak{M}_{1}+\mathfrak{N}_{2}}\right)= \\
& \frac{2 \pi \rho_{1} D_{12}}{\mathfrak{N}_{1}+\mathfrak{N}_{2}}\left[\frac{d \log w}{d \log r}-\left(\frac{m_{2}}{m_{1}}-1\right) \frac{v^{2}}{R T}\right]
\end{aligned}
$$

The most important features of the vortex containment mechanism can be seen quite readily from Eq. (7). Suppose first that there is no radial flow, so that $\mathfrak{N}_{1}=\mathfrak{M}_{2}=0$; then the quantity in square brackets must be zero, and we have

$$
\log w=\left(\frac{m_{2}}{m_{1}}-1\right) \int\left(\frac{v^{2}}{R T}\right) \frac{d r}{r}+\text { constant }
$$

Thus, $w$ increases monotonically with $r$ for $m_{2} / m_{1}>1$. This is of course the equilibrium distribution for a given variation of $v$ and $T$ with $r$.

Next, suppose that $\mathfrak{M}_{1}$ and $\mathfrak{N}_{2}$ each have some negative value, but that $\left|\mathscr{T T}_{2}\right| \ll\left|\mathscr{T}_{1}\right|$; then, except where $w$ is very small, the left side of Eq. (7) is unity, and except for the variation of the quantity $2 \pi \rho_{1} D_{12} /$ $\left(\mathscr{M}_{1}+\mathfrak{M}_{2}\right)$, the sum of the two terms in brackets must be a negative constant. Thus, initially as $\mathfrak{M}_{1}$ decreases from zero, $d w / d r$ must decrease. If $\mathfrak{T}_{1}$ is made sufficiently negative, $d w / d r$ can be made zero at any given 
value of $r$. Now, for such a value of $\mathfrak{M T}_{1}$, if $v^{2} / R T$ decreases monotonically with increasing $r, d w / d r$ will be positive for $r$ less than the point at which $d w / d r$ is zero, and negative for $r$ larger than this value. Thus, a simple maximum is produced.

The position of this maximum may be obtained by solving Eq. (7) with $d w / d r=0$. If $M_{t m}=v /(\gamma R T)^{1 / 2}$ is the tangential Mach number at the point of the maximum, and other quantities evaluated at the maximum are similarly denoted by subscript $m$, then

$$
\begin{aligned}
\gamma M_{t m}^{2}=\frac{-1}{\left(m_{2} / m_{1}\right)}-1\left(\frac{\mathscr{N}_{1}+\mathfrak{M}_{2}}{2 \pi \rho_{1} D_{12}}\right)_{m} \times \\
{\left[1-\left(\frac{1+w_{m}}{w_{m}}\right)\left(\frac{\mathfrak{M}_{2}}{\mathfrak{M}_{1}+\mathfrak{N}_{2}}\right)\right] }
\end{aligned}
$$

Eq. (8) contains one of the most important pieces of information to be derived from the analysis, namely, that the radial mass flow permitted by the diffusion process is independent of the diameter of the vortex, and in fact depends only on $\mathfrak{M}_{2} / \mathfrak{T T}_{1}, \gamma, M_{t_{m}}{ }^{2}$ and the product $\left(\rho_{1} D_{12}\right)_{m}$ evaluated at the maximum point of $w$, since for the cases of interest $\mathfrak{M}_{2} /\left(\mathfrak{M}_{1}+\mathfrak{M}_{2}\right) \ll$ $w_{m} /\left(1+w_{m}\right)$. For a given pair of gases, $\left(\rho_{1} D_{12}\right)_{m}$ depends only on $T_{m}$. The most important fact is that the mass flow rate per unit of vortex length is independent of the vortex radius. Physically, this is a result of the fact that for a fixed Mach number, the pressure gradient is inversely proportional to the vortex radius, and the resulting diffusion velocity is therefore also inversely proportional to the radius of the vortex. But the mass flow rate per unit of vortex length is proportional to the product of the radial velocity (which is essentially the diffusion velocity) and the vortex radius, and hence is constant.

Finally, if $v^{2} / R T$ is not monotonic but first decreases and then increases with increasing $r, w$ can have both a maximum and a minimum. It will be demonstrated that this situation can occur with heat addition in a free vortex. There will then be two positions in the vortex where $d w / d r=0$. In a free vortex, where $v \propto 1 / r$, there will be a level of heat addition below which only a simple maximum will exist because $T$ does not vary rapidly enough to overcome the variation of $v$. This value will be termed the critical rate of heat addition. There will also be a larger rate of heat addition above which no maximum of $w$ can exist, because $\left(v^{2} / R T\right)$ increases monotonically with increasing $r$. This value will be termed the maximum rate of heat addition. Both of these values are of course dependent on $2 \pi \rho_{1} D_{12} /\left(\mathfrak{T}_{1}+\mathfrak{M}_{2}\right), \mathfrak{M}_{2} / \mathfrak{I T}_{1}, m_{2} / m_{1}$, and $M_{t m}$.

\section{The Energy Equation}

To make these relationships quantitative, we must consider the energy equation, since it describes the behavior of $T$. The viscous dissipation will be neglected, as it is quite negligible in a flow with large heat addition. The equation in terms of the enthalpy $h$ is then ${ }^{3}$

$$
\rho u \frac{d h}{d r}=u \frac{d p}{d r}-\frac{1}{r} \frac{d}{d r}(r q)+g
$$

where $q=\lambda(d T / d r)+\left(C_{p 2}-C_{p 1}\right) \rho_{2} u_{2} T, \lambda$ being the heat conductivity, and $g$ is the volumetric rate of heat addition. In this case, $g$ of course represents the heating by fission fragments.

The heating rate is proportional to the concentration, $n_{2}$, of fissionable material, to its fission cross section, $\sigma_{f}$, and to the neutron flux, $\phi$. If the local energy release per fission is $Q$, then $g=Q \sigma_{f} \phi \eta_{2}$. In the present work, it will be assumed that $\sigma_{\rho}$ and $\phi$ are independent of $r . \quad Q$ is a constant for practical purposes, so the heating rate is simply proportional to $n_{2}$. $h$ is the enthalpy of the gas mixture, and under the assumption that $n_{2} \ll n$, it can be written as $h=C_{p 1} T /$ $(1+w)$. The specific heat of the light gas will be taken constant, although this is a very crude assumption for diatomic gases at the temperatures which are

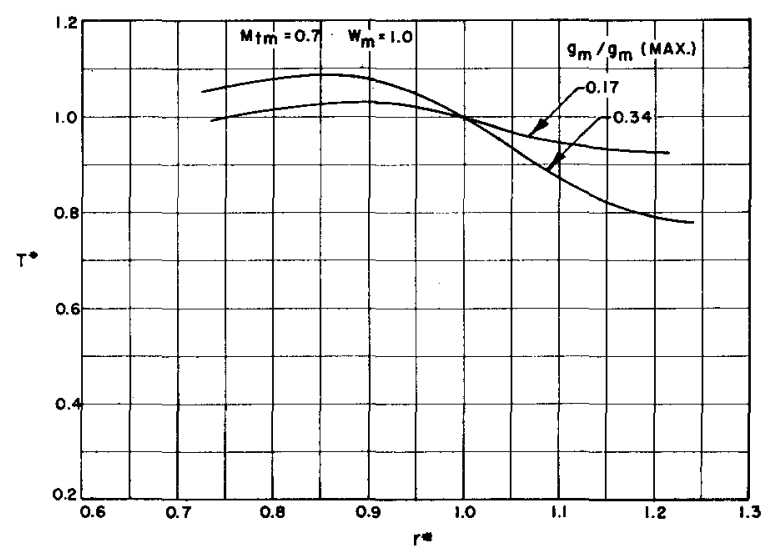

FIG. 3. Variation of dimensionless temperature, $T^{*}$, with dimensionless radius, $r^{*}$, for $g_{m}$ less than $g_{m}$ (crit).

of interest for the gaseous fission rocket. We make the assumption in order that the calculations shall not depend explicitly on the temperature and pressure level, as they would if the exact relationship between $h$ and $T$ were included. With the expression previously given for $d p / d r$, Eq. (9) then becomes

$$
\begin{aligned}
\frac{d}{d r}\left(\frac{C_{p 1} T}{1+w}\right) & =\frac{v^{2}}{r}-\frac{2 \pi}{\mathfrak{M}_{1}+\mathfrak{T}_{2}} \times \\
\frac{d}{d r} & {\left[\lambda r \frac{d T}{d r}+\left(C_{p 2}-C_{p 1}\right) r \rho_{2} u_{2} T\right]+Q \sigma_{f} \phi n_{2} / \rho u }
\end{aligned}
$$

From Eq. (2),

$\rho_{2} u_{2} r=$

$$
\left[\left(\mathfrak{M}_{1}+\mathfrak{M}_{2}\right) / 2 \pi\right]\left[\mathfrak{M}_{2} /\left(\mathfrak{M}_{1}+\mathfrak{M}_{2}\right)+w /(1+w)\right]
$$

and from Eq. (1),

$Q \sigma_{f} \phi n_{2} / \rho u=\left[2 \pi Q \sigma_{f} \phi / m_{2}\left(\mathfrak{T}_{1}+\mathfrak{T}_{2}\right)\right] r \rho_{1} w$

so that 


$$
\begin{aligned}
& \frac{d}{d r}\left(\frac{T}{1+w}\right)=\frac{v^{2}}{C_{p 1} r}+\left(1-\frac{C_{p 2}}{C_{p 1}}\right) \times \\
& \frac{d}{d r}\left[\left(\frac{\mathfrak{M}_{2}}{\mathfrak{M}_{1}+\mathfrak{N}_{2}}-\frac{w}{1+w}\right) T\right]-\frac{2 \pi}{\mathfrak{N}_{1}+\mathfrak{N}_{2}} \times \\
& \quad \cdot \frac{d}{d r}\left(\frac{\lambda}{C_{p 1}} r \frac{d T}{d r}\right)+\frac{2 \pi Q \sigma_{f} \phi}{m_{2} C_{p 1}\left(\mathfrak{N}_{1}+\mathfrak{N} C_{2}\right)} r \rho_{1} w
\end{aligned}
$$

It will be helpful to consider the relative magnitudes of the heat transfer by conduction and diffusion. Assuming that $r d T / d r$ and $T$ are of the same order, the ratio of the conductive and diffusive heat transfer rates is $\left[2 \pi \lambda / C_{p 1}\left(\mathscr{T}_{1}+\mathfrak{M}_{2}\right)\right] /[w /(1+w)]$, at points where $w \gg \mathfrak{K}_{2} / \mathfrak{N}_{1}$. Now according to Euchen's formula, ${ }^{3}$ the heat conductivity, viscosity, and specific heat are related by $\lambda=(9 \gamma-5) \mu C_{v} / 4$, so that the ratio becomes

$$
\frac{9 \gamma-5}{4 \gamma}\left(\frac{1+w}{w}\right)\left(\frac{2 \pi \mu}{\mathfrak{M}_{1}+\mathfrak{M}_{2}}\right)
$$

It will become clear later that $w$ must be of the order of unity or greater over most of the vortex to provide sufficient fissionable material for nuclear criticality, so that the factor $(1+w) / w$ is of order unity. The factor $\left(\mathfrak{M}_{1}+\mathfrak{N}_{2}\right) / 2 \pi \mu$ is the radial Reynolds number of the flow; for the situations of interest it is of the order of 100 . Thus, conduction can be neglected compared to diffusive energy transport, except where $r(d T / d r) \gg T$.

Neglecting the conduction term, carrying out the indicated differentiation, and eliminating $d w / d r$ with Eq. (7), we find the following expression for $(d \log T) \div$ $(d \log r)$ :

$$
\begin{aligned}
& \frac{d \log T}{d \log r}=\frac{\frac{1+w}{w}\left[(\gamma-1) M^{2}+\frac{2 \pi Q \sigma_{f} \phi r^{2} \rho_{1} w}{m_{2} C_{p 1}\left(\mathfrak{M}_{1}+\mathfrak{M}_{2}\right) T}\right.}{\frac{1}{w}+\left(1-\frac{C_{p 2}}{C_{p 1}}\right)\left[1-\left(\frac{1+w}{w}\right)\left(\frac{\mathfrak{M}_{2}}{\mathfrak{M}_{1}+\mathfrak{M}_{2}}\right)\right]}+ \\
& \frac{C_{p 2}}{C_{p 1}}\left(\frac{1}{1+w}\right)\left\{\frac{\mathfrak{N}_{1}+\mathfrak{N}_{2}}{2 \pi \rho_{1} D_{12}}\left[1-\left(\frac{1+w}{w}\right)\left(\frac{\mathfrak{N}_{2}}{\mathfrak{M}_{1}+\mathfrak{M}_{2}}\right)\right]+\left(\frac{m_{2}}{m_{1}}-1\right) \gamma M^{2}\right\} \\
& \frac{1}{w}+\left(1-\frac{C_{p 2}}{C_{p 1}}\right)\left[1-\left(\frac{1+w}{w}\right)\left(\frac{\mathfrak{M}_{2}}{\mathfrak{M}_{1}+\mathfrak{M}_{2}}\right)\right]
\end{aligned}
$$

From Eq. (7), the accompanying expression for $(d \log w / d \log r)$ is

$$
\frac{d \log w}{d \log r}=\left(\frac{\mathfrak{M}_{1}+\mathfrak{M}_{2}}{2 \pi \rho_{1} D_{12}}\right)\left[1-\left(\frac{1+w}{w}\right)\left(\frac{\mathfrak{M}_{2}}{\mathfrak{M}_{1}+\mathfrak{M}_{2}}\right)\right]+\left(\frac{m_{2}}{m_{1}}-1\right) \gamma M^{2}
$$

and from Eq. (6),

$$
(d \log p) /(d \log r)=\gamma M^{2}(1+w)
$$

With the proper boundary conditions, and the relation $\rho_{1}=p / R T$, these three first-order nonlinear equations may be solved for $T$, $w$, and $p$, if $v$ is given as a function of $r$. As was mentioned in the introduction, it will be assumed in the present work that $v \propto 1 / r$, i.e., that the flow is inviscid.

Only those solutions yielding a maximum of $w$ within the vortex will be considered. For heating rates below the critical heating rate, such solutions offer the possibility of containing the fissionable material within the vortex, away from the outer wall. For heating rates above the critical value, the value of $w$ at the outer boundary of the vortex will be higher, in contrast.

To facilitate the numerical integration, it is desirable to rewrite the equations in terms of dimensionless variables. A particularly convenient set of variables is obtained by dividing each of the variables by its value at the radius, $r_{m}$, where $w$ is a maximum. Such variables will be denoted by an asterisk-e.g., $r^{*}=r / r_{m}, T^{*}=T / T_{m}$. It is also convenient to introduce a dimensionless heating rate $g_{m}$, evaluated at $r_{m}$, as

$$
g_{m}=-\left[2 \pi Q \sigma_{f} \phi r_{m}{ }^{2} n_{2 m}\right] /\left[C_{p 1}\left(\mathfrak{N}_{1}+\mathfrak{M}_{2}\right) T_{m}\right]
$$

Through Eq. (8), $\left(\mathfrak{M}_{1}+\mathfrak{M}_{2}^{\prime}\right) / 2 \pi \rho_{1} D_{12}$ may be related to $M_{t_{m}}, w_{m}$, and $\mathfrak{M}_{2} /\left(\mathfrak{K}_{1}+\mathfrak{T}_{2}\right)$, provided the dependence of $D_{12}$ on the state of the gas mixture is specified. For the present purposes, the simple result for $D_{12}$ based on hardsphere collisions, namely,

$$
D_{12}=\left(3 / 8 n d_{12}^{2}\right)\left[\left(m_{1}+m_{2}\right) k T / 2 \pi m_{1} m_{2}\right]^{1 / 2}
$$

is adequate. $k$ is Boltzmann's constant and $d_{12}$ is the effective collision diameter. Thus, $D_{12} /\left(D_{12}\right) m=T^{* 3 / 2} / p^{*}$, and

$$
\frac{\mathfrak{M}_{1}+\mathfrak{M}_{2}}{2 \pi \rho_{1} D_{12}}=\left(\frac{\mathfrak{M}_{1}+\mathfrak{M}_{2}}{2 \pi \rho_{1} D_{12}}\right)_{m} T^{*-1 / 2}=-\frac{\gamma M_{m_{m}^{2}}\left[\left(m_{2} / m_{1}\right)-1\right] T^{*-1 / 2}}{1-\left(\frac{1+w_{m}}{w_{m}}\right)\left(\frac{\mathfrak{M}_{2}}{\mathfrak{N}_{1}+\mathfrak{M}_{2}}\right)}
$$

With these relations. Eq. (12) becomes 


$$
\begin{aligned}
\frac{d \log T^{*}}{d \log r^{*}}=\frac{\frac{1+w}{w}\left[\frac{(\gamma-1) M_{m^{2}}}{r^{* 2} T^{*}}-g m \frac{r^{* 2} p^{*} w}{T^{* 2} w_{m}}\right]}{\frac{1}{w}+\left(1-\frac{C_{p 2}}{C_{p 1}}\right)\left[1-\left(\frac{1+w}{w}\right)\left(\frac{\mathfrak{N}_{2}}{\mathfrak{M}_{1}+\mathfrak{M}_{2}}\right)\right]}+ \\
\frac{\frac{\gamma M_{m^{2}}{ }^{2}\left[\left(m_{2} / m_{1}\right)-1\right]\left(C_{p 2} / C_{p 1}\right)}{(1+w) T^{* 1 / 2}}\left[\frac{1}{r^{* 2} T^{* 1 / 2}}-\frac{1-\left(\frac{1+w}{w}\right)\left(\frac{\mathfrak{M}_{2}}{\mathfrak{M}_{1}+\mathfrak{M}_{2}}\right)}{\left.1-\left(\frac{1+w_{m}}{w_{m}}\right)\left(\frac{\mathfrak{M}_{2}}{\mathfrak{M}_{1}+\mathfrak{M}_{2}}\right)\right]}\right.}{\frac{1}{w}+\left(1-\frac{C_{p 2}}{C_{p 1}}\right)\left[1-\left(\frac{1+w}{w}\right)\left(\frac{\mathfrak{M}_{2}}{\mathfrak{M}_{1}+\mathfrak{M}_{2}}\right)\right.}
\end{aligned}
$$

Similarly, Eq. (13) is

$$
\frac{d \log w}{d \log r^{*}}=\frac{\gamma M_{t m}^{2}\left[\left(m_{2} / m_{1}\right)-1\right]}{T^{* 1 / 2}}\left[\frac{1}{r^{*} T^{* 1 / 2}}-\frac{1-\left(\frac{1+w}{w}\right)\left(\frac{\mathfrak{M}_{2}}{\mathfrak{M}_{1}+\mathfrak{M}_{2}}\right)}{1-\left(\frac{1+w_{m}}{w_{m}}\right)\left(\frac{\mathfrak{M}_{2}}{\mathfrak{M}_{1}+\mathfrak{M}_{2}}\right)}\right]
$$

and Eq. (14) reduces to

$$
\left(d \log p^{*}\right) /\left(d \log r^{*}\right)=\gamma M_{t m}{ }^{2}(1+w) / r^{* 2} T^{*}
$$

\section{Boundary Conditions}

The system of three first-order differential equations, (18), (19), and (20), allows one boundary condition to be satisfied on each of the three variables $w, T^{*}$, and $p^{*}$. In addition, the quantity $\mathfrak{M}_{2} /\left(\mathfrak{M}_{1}+\mathfrak{M}_{2}\right)$ is a characteristic value of the diffusion equation, as will be demonstrated. It is of course clear that by neglecting conduction in the energy equation Eq. (18) is reduced from a second-order equation to a first-order equation; this reduces the number of applicable boundary conditions to one. In a vortex such as is sketched in Fig. 1, the boundary condition on $T$ at the vortex tube surface, namely, that the gas temperature equals the surface temperature, is lost. Similarly, by neglecting the radial acceleration in the radial momentum equation, Eq. (20) is reduced to a first-order equation. If the pressure is specified at any radius in a tube such that in Fig. 1, Eq. (20) appears to determine the pressure everywhere else. Actually, the three-dimensional flow near the axis of such a tube would be an important factor in determining the pressure level in the tube.

The situation is perhaps not so clear in the case of the diffusion equation. If, for example, the vortex is assumed to be bounded at its inner and outer radii by porous cylinders, it may appear that the value of the density ratio, $w$, in the vortex just adjacent to the surface of each of the porous cylinders should equal the value of $w$ in the gas mixture flowing through the porous cylinder. However, this is only true if the direction of gas flow is from the vortex to the porous surface, i.e., if the boundary is an outflow boundary. At such a boundary, the mass flow rate of each species is proportional to its concentration. The inner boundary of the present vortex flow is such a boundary; if the radius of this inner boundary is $r_{\mathfrak{c}}$, and the corresponding value of $w$ is $w_{c}$, then it is clear that the quan- tity $\mathfrak{N}_{2} /\left(\mathfrak{N}_{1}+\mathfrak{M}_{2}\right)$, which is the ratio of heavy gas mass flow to total mass flow, is simply

$$
\mathfrak{M}_{2} /\left(\mathfrak{M}_{1}+\mathfrak{M}_{2}\right)=w_{c} /\left(1+w_{c}\right)
$$

Thus, the characteristic value of the diffusion equation is determined at the inner boundary.

In contrast, all that is required at the outer, or inflow, boundary is that the gas flowing into the vortex have the same $w$ as that leaving, namely, $w_{c}$. It is neither necessary nor in general possible for the value of $w$ in the vortex adjacent to the inflow boundary to equal $w_{c}$. The transition from one value of $w$ to the other at the inflow boundary occurs by a mixing process, and results in an increase of the entropy of the gas mixture. This entropy increase due to mixing is offset by the separative effect in the vortex, so that the entropies of mixing of the inlet and outlet flows are equal.

For the present calculations, the boundary conditions will be applied at $r^{*}=1$, and are simply

$$
T^{*}(1)=1, \quad p^{*}(1)=1, \quad w(1)=w_{m}
$$

where $w_{m}$ is the maximum value of $w$. For given values of $g_{m}, w_{c}, w_{m}, M_{t m}, m_{2} / m_{1}, C_{p 2} / C_{p 1}$, and $\gamma$, the solution can then be obtained by forward integration. The inner radius of the vortex is then defined as that radius at which $w$ is equal to $w_{c}$.

The last statement implies that the fluid leaves the vortex at the radius $r_{c}$, as though there were a cylindrical sink there. This condition would be approximated by a porous cylinder of radius $r_{c}$. The threedimensional flow near the axis of a tube such as that sketched in Fig. 1 would act as a sink distributed over the portion of the tube volume within the radius of the exit nozzle. For a precise evaluation of the loss of fissionable material from the vortex, a treatment of this 


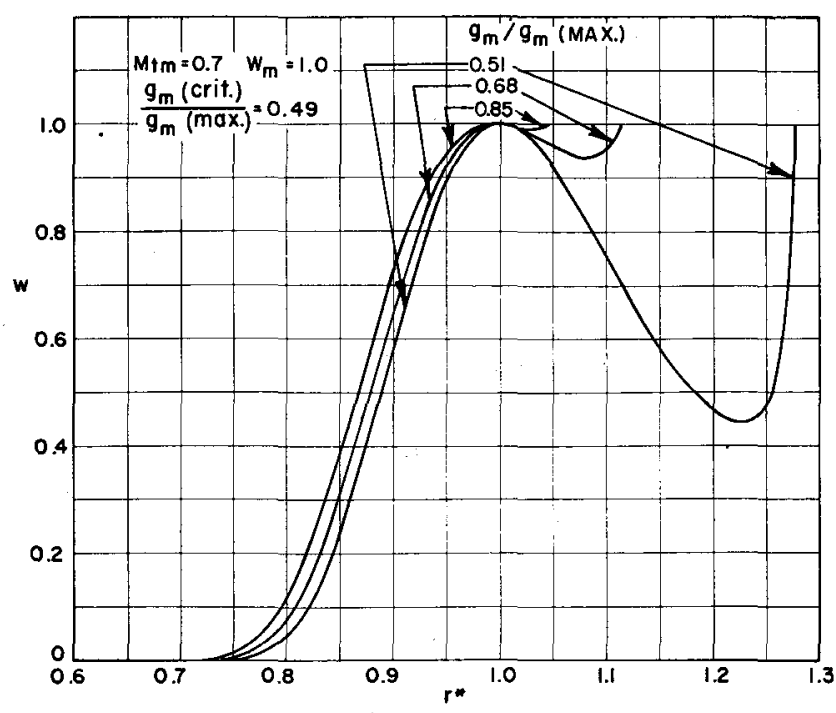

FIG. 4. Variation of density ratio, $w$, with dimensionless radius, $r^{*}$, for $g_{m}$ greater than $g_{m}$ (crit).

distributed sink would be essential; for the present purpose, $w_{c}$ will be taken to be very small $\left(10^{-4}\right)$, and the fluid will be assumed to leave the vortex at $r_{c}$. This gives a very conservative estimate of the loss rate.

Finally, it should be noted that for $w_{c} / w_{m} \ll 1$, the character of the solutions for $T^{*}, p^{*}$, and $w$ is independent of the magnitude of $w_{c}$ [see Eq. (19)].

\section{The Critical and Maximum Heating Rates}

The maximum heating rate has been defined as the highest heating rate for which $w$ has a maximum within the vortex. Equivalently, $g_{m(\max )}$ is the largest value of $g_{m}$ for which $w$ has a maximum at $r^{*}=1$; that is, the largest value of $g_{m}$ for which $d^{2} w / d r^{* 2}$ is negative at $r^{*}=1$. From Eq. (19),

$$
\begin{aligned}
\left(\frac{d^{2} w}{d r^{* 2}}\right)_{r^{*}=1}=-\gamma M_{t m}^{2}\left(\frac{m_{2}}{m_{1}}-1\right) \times \\
\\
w_{m}\left[2+(1 / 2)\left(d T^{*} / d r^{*}\right)_{r^{*}=1}\right]
\end{aligned}
$$

so that for $g_{m(\max )},\left(d I^{*} / d r^{*}\right)_{r *=1}=-4$. Now from Eq. (18),

$$
\left(\frac{d T^{*}}{d r^{*}}\right)_{r^{*}=1}=\frac{\frac{1+w_{m}}{w_{m}}\left[(\gamma-1) M_{t m}{ }^{2}-g_{m}\right]}{\frac{1}{w_{m}}+\left(1-\frac{C_{p 2}}{C_{p 1}}\right)\left[\frac{1-\left(w_{c} / w_{m}\right)}{1+w_{c}}\right]}
$$

whence

$$
\begin{aligned}
& g_{m(\max )}=(\gamma-1) M_{t m}{ }^{2}+ \\
& 4\left[\frac{1}{w_{m}}+\left(1-\frac{C_{p 2}}{C_{p 1}}\right)\left(\frac{1-\left(w_{c} / w_{m}\right)}{1+w_{c}}\right)\right]\left(\frac{w_{m}}{1+w_{m}}\right)
\end{aligned}
$$

and for $w_{c} \ll 1$ and $w_{c} \ll w_{m}$, this is

$$
g_{m(\max )}=4+(\gamma-1) M_{t m^{2}}
$$

The critical heating rate has been defined as the smallest heating rate for which $d w / d r^{*}=0$ for some $r^{*}>1$. From Eq. (19), for $w_{c} \ll w_{m}, d w / d r^{*}$ is zero if $T^{*}=1 / r^{* 4}$; however, since $T^{*}$ can be obtained only from integration of the differential equations, the value of $g_{m(c r i t)}$ cannot readily be obtained analytically. The values of $g_{m(\text { crit })} / g_{m(\max )}$ obtained from the solutions of the equations will be given later.

\section{Solution of the Diffusion Problem}

Solutions of a system of equations equivalent to Eqs. (18), (19), and (20) with boundary conditions (22) were given in reference 1 . These were obtained by forward integration of the equations, from $r^{*}=1$ to both smaller and larger $r^{*}$. The parameters which characterize the gas mixture, and which occur explicitly in Eqs. (18), (19), and (20), were chosen to be representative of hydrogen and plutonium gas. Specifically, $m_{2} / m_{1}=119.5, C_{p 2} / C_{r 1}=0.008$, and $\gamma=$ 1.31 were chosen. The exit density ratio, $w_{c}$, was taken as 0.0001 .

The remaining parameters are then $g_{m}$, which measures the heating rate; $M_{t m}$, which measures the strength of the vortex; and $w_{m}$, which is a measure of the density ratio of heavy gas contained in the vortex. While the specification of $w_{c}$ effectively determines the inner boundary of the vortex, the value of $r^{*}$ corresponding to the outer boundary is arbitrary. The numerical solutions must, however, be terminated for some $r^{*}$ greater than unity. In the calculations reported in reference 1 , they were terminated when the density ratio $w$ reached $w_{m} / 10$ for $g_{m}<g_{m(c r i t),}$, and when $w$ reached $w_{m}$, for $g_{m}>g_{m(c r i t)}$.

\section{Solutions for $w$ and $T^{*}$}

Solutions for $w$ and $T^{*}$, for two subcritical values of the heating parameter, are shown in Figs. 2 and 3. The heating rates are $0.17 g_{m(\max )}$ and $0.34 g_{m(\max )}$. For the chosen values of $M_{t m}=0.7$ and $w_{m}=1.0, g_{m(c r i t)} /$ $g_{m(\max )}=0.49$. In this range of $g_{m} / g_{m(\max )}$, the $w$ profile is rather insensitive to $g_{m}$, even though a considerable amount of heating takes place. If $g_{m}$ were zero, $T^{*}$ would decrease quite rapidly with decreasing $r^{*}$, because of the expansion in the pressure gradient pro-

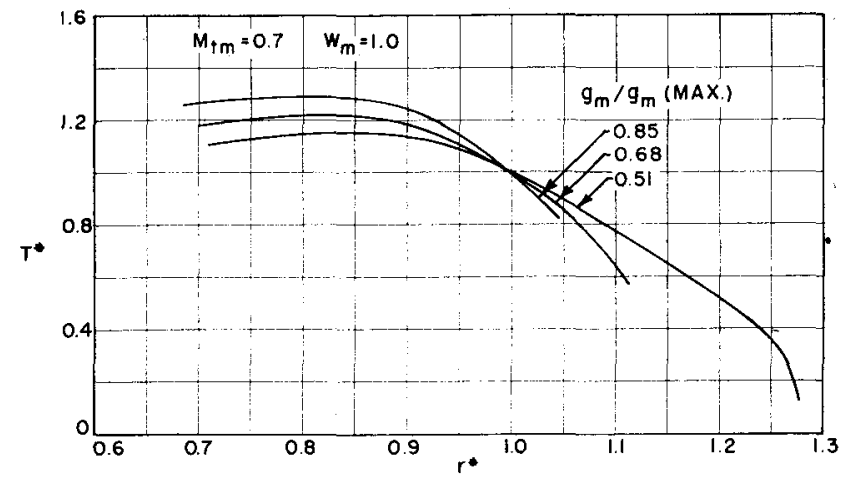

FIG. 5. Variation of dimensionless temperature, $T^{*}$, with dimensionless radius $r^{*}$; for $g_{m}$ greater than $g_{m}$ (crit). 


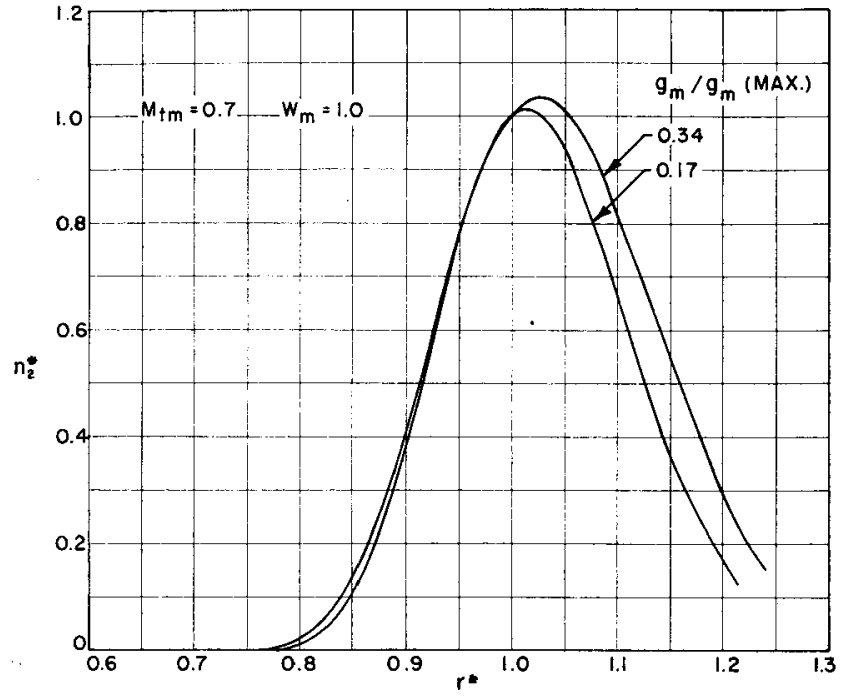

FIG. 6. Variation of dimensionless fuel concentration, $n_{2}{ }^{*}$, with dimensionless radius, $r^{*}$, for $g_{m}$ less than $g_{m}$ (crit.)

duced by the vortex. It follows that for sufficiently large $r^{*}, T^{*}$ would increase with $r^{*}$. In Figs. 2 and 3 , the solutions are carried out approximately to the value of $r^{*}$ at which $d T^{*} / d r^{*}=0$.

As $g_{m}$ increases, $d T^{*} / d r^{*}$ increases until the critical value, $g_{m(c r i t)}$, is reached. The solutions then change character to that shown in Figs. 4 and 5. The essential differences are that $w$ has a second stationary point for some $r^{*}>1$, and that $d T^{*} / d r^{*}$ becomes increasingly negative with increasing $r^{*}$, instead of eventually becoming positive, as in Fig. 3 . The behavior appears to be singular, with $w$ approaching infinity and $T^{*}$ approaching zero as $r^{*}$ approaches some value. Thus, in principle, very large temperature ratios, from the outside of the vortex to the inside, can be obtained with sufficiently large heating rates.

However, the irregular behavior of $w$ and $T^{*}$ implies very large concentrations of heavy gas in the outer portions of the vortex tube. This is illustrated in Figs. 6 and 7 , which give the profiles of $n_{2}{ }^{*}$ corresponding to the $w$ and $T^{*}$ profiles of Figs. 2 and 3 , and 4 and 5 , respectively. For the subcritical heating rates, the profiles of $n_{2}{ }^{*}$ and $w$ are at least qualitatively similar; however, for the supercritical heating rates, $n_{2}{ }^{*}$ becomes very large in the outer portions of the tube. This is, of course, principally a reflection of the fact that the gas density is much larger in the outer portions because of the large temperature change.

Values of $g_{m(c r i t)} / g_{m(\max )}$ obtained from a series of integrations are summarized in Table 1.

The shatpness of the peak in the $w$ distribution increases rapidly with increasing $M_{t m}$, as shown in Fig. 8 for three cases having $g_{m} / g_{m(\text { crit })} \approx 1.02$. Thus, as

TABLE 1

\begin{tabular}{ccccc}
\hline \multicolumn{5}{c}{ Critical Heating Rates } \\
\hline$M_{i m}$ & $w_{m}=0.2$ & $w_{m}=0.5$ & $w_{m}=1.0$ & $w_{m}=4.0$ \\
\hline 0.5 & 0.455 & 0.450 & 0.441 & 0.410 \\
0.7 & 0.505 & 0.499 & 0.487 & 0.434 \\
1.0 & 0.580 & 0.574 & 0.557 & 0.470 \\
\hline
\end{tabular}

the Mach number inicreases, the amount of heavy gas which is retained in the vortex, for a fixed value of $w_{m}$, is reduced. On the other hand, increasing $M_{t m}$ increases the mass flow capacity of the vortex, as may be seen from Eq. (8).

In contrast to the strong dependence of the profiles of $w$ on $M_{t m}$, their dependence on $w_{m}$ is quite weak. $w / w_{m}$ is shown in Fig. 9 for three values of $w_{m}$, and for $g_{m} / g_{m(c r i t)} \approx 1.02$, as before. This insensitivity of $w / w_{m}$ to $w_{m}$ may be understood from a careful examination of Eqs. (18) and (19). If we consider only the range of $w$ where $w /(1+w) \gg \mathfrak{M}_{2} /\left(\mathfrak{M}_{1}+\mathfrak{M}_{2}\right)$, they reduce approximately to:

$$
\begin{aligned}
& \frac{d \log T^{*}}{d \log r^{*}}= \\
& \gamma M_{t m}^{2}\left(\frac{m_{2}}{m_{1}}-1\right) \frac{C_{p 2}}{C_{p 1}} \frac{w}{(1+w)^{2}}\left(\frac{1}{r^{* 2} T^{* 1 / 2}}-1\right)+ \\
& \frac{(\gamma-1) M_{t m}^{2}}{r^{* 2} T^{*}}-g_{m} \frac{r^{* 2} p^{*}}{T^{* 2}}\left(\frac{w}{w_{m}}\right)
\end{aligned}
$$

and

$$
\frac{d \log w}{d \log r^{*}}=\frac{\gamma M_{t m}^{2}}{T^{* 1 / 2}}\left(\frac{m_{2}}{m_{1}}-1\right)\left(\frac{1}{r^{* 2} T^{* 1 / 2}}-1\right)
$$

In the energy equation, the first term represents the energy transport by diffusion, and this term depends directly on the magnitude of $w$; however, it is zero at two points (the maximum and minimum of $w$ ) on the curves of Fig. 9, so it seems reasonable that it is fairly small over most of the vortex. Of the other two terms, the last depends on $w / w_{m}$, and not on $w_{m}$ itself. Thus, it may be said that the profiles of $w / w_{m}$ are similar for different values of $w_{m}$ because the diffusive energy transport is comparatively unimportant.

\section{Performance Parameters}

In addition to the three parameters determining the solutions, namely, $M_{t m}, w_{m}$, and $g_{m}$, the value of $r^{*}$ cor-

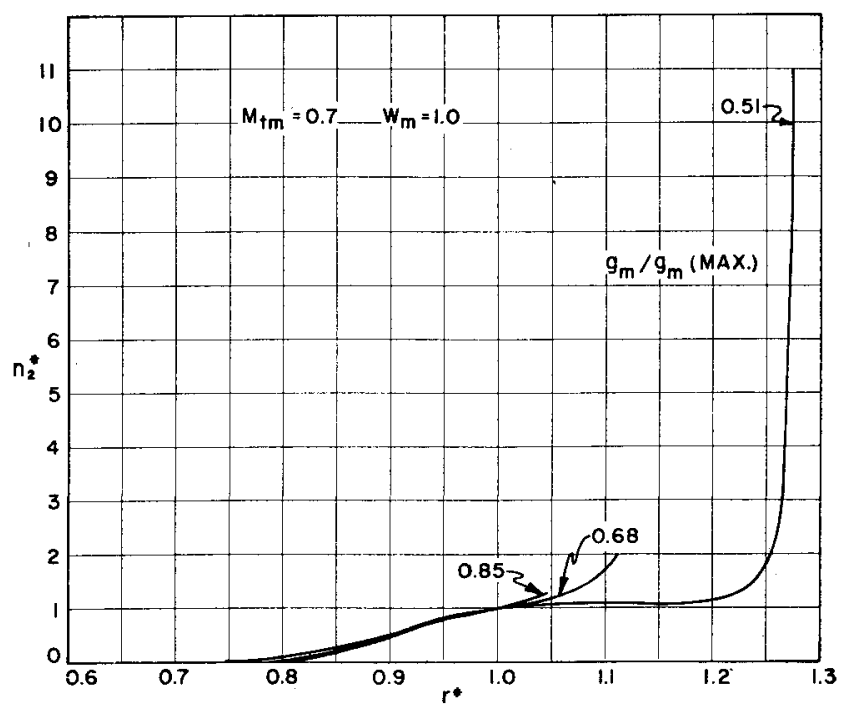

FIG. 7. Variation of dimensionless fuel concentration, $n_{2}{ }^{*}$, with dimensionless radius, $r^{*}$, for $g_{m}$ greater than $g_{m}$ (crit). 
responding to the periphery of the vortex must be specified in describing an actual vortex. For given values of $M_{t_{m}}, w_{m}$, and $g_{m}$, the pressure and temperature ratios across the vortex, for example, depend on the value of $r_{p}{ }^{*}$. In order to reduce the number of parameters by one, a rather arbitrary choice of $r_{p}{ }^{*}$ will therefore be made. For the cases with subcritical heating rates, $r_{p}{ }^{*}$ will be taken as that value of $r^{*}$ greater than unity for which $w / w_{m}=0.1$. For the supercritical cases $r_{p}{ }^{*}$ will be taken as the value of $r^{*}$ greater than unity for which $w=w_{m}$.

With these choices, there is then, for given values of $w_{m}$ and $M_{t m}$, a definite relationship between the temperature ratio across the vortex, $T_{c} / T_{p}$, and the heating parameter. This relationship is shown in Fig. 10 for $M_{t m}=0.7$. It will be noted that the characteristics are markedly different for the subcritical and supercritical solutions. As the heating parameter is increased, the temperature ratio, $T_{c} / T_{p}$, increases continuously until $g_{m(c r i t)}$ is reached. At this point, the profile of $w$ switches from the type shown in Fig. 2 to that shown in Fig. 4, and the temperature ratio becomes very large. As $g_{m}$ is increased beyond $g_{m(c r i t)}, T_{c} / T_{p}$ decreases again, because with the above choice for the outer radius, $r_{p}{ }^{*}$ decreases with increasing $g_{m}$. For $g_{m} / g_{m(\max )}=1$, the outer boundary of the vortex is at the maximum of $w$, i.e., at $r^{*}=1$. Because of the sensitivity of the profiles in the neighborhood of $g_{m(c r i t)}$, it was rather difficult to establish the exact end points of the subcritical characteristics; hence, these end points are labeled approximate. For $M_{t m}=$ 0.7 , the maximum values of $T_{c} / T_{p}$ which can be obtained with a simple maximum of $w$ vary from about 3.0 for $w_{m}=0.2$ to 2.5 for $w_{m}=4.0$. Values for other Mach numbers are given in Table 2 .

It is convenient to relate other performance parameters to the temperature ratio, $w_{m}$, and $M_{t m}$. The pressure ratio from the inner to the outer boundary is shown in Fig. 11 for $M_{t m}=0.7$. As $w_{m}$ increases, the pressure drop through the vortex increases also. This increase may be regarded as due to the increased resistance to flow offered by the more dense distribution of heavy gas as $w_{m}$ increases.

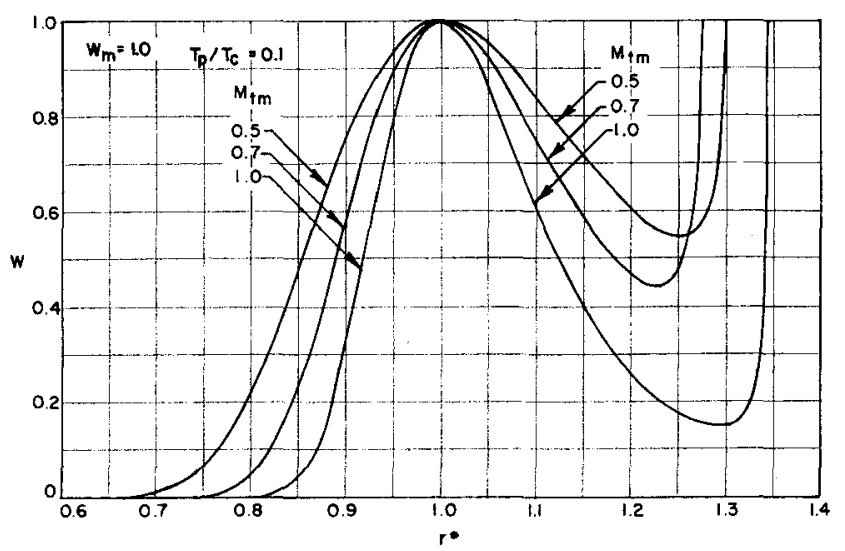

Fig. 8. Effect of the tangential Mach number at the point of maximum density ratio, $M_{t m}$, on the variation of the density ratio, $w$, with dimensionless radius, $r^{*}$.

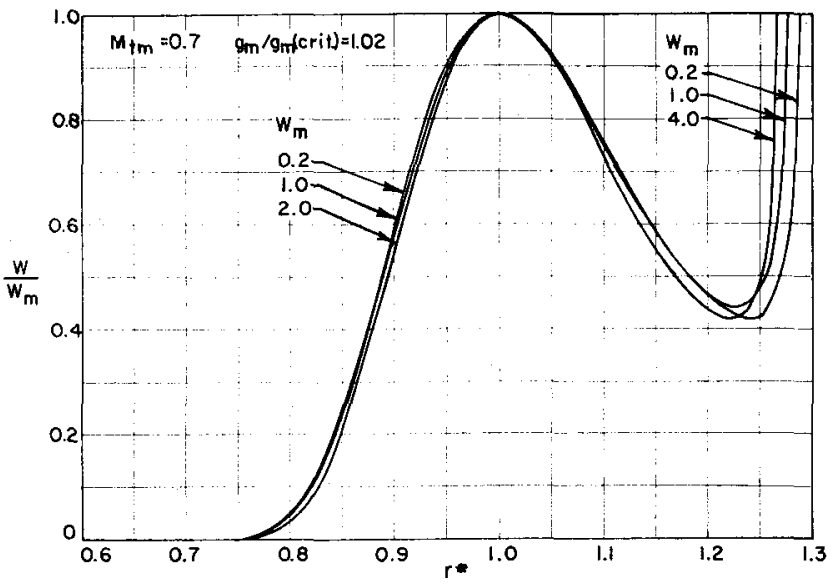

Fig. 9. Effect of the maximum density ratio, $w_{m}$, on the variation of the density ratio, $w$, with the dimensionless radius, $r^{*}$.

The Mach number of the vortex at its periphery, $M_{t p}$, is related to $M_{t m}$ by

$$
M_{t p}=M_{t m} / r_{p}{ }^{*} T_{p}{ }^{* 1 / 2}
$$

Since $w / w_{m}$ and $T^{*}$ were found to be independent of $w_{m}$ for given $M_{t m}$, it is not surprising that, as a function of $T_{c} / T_{p}, M_{t p}$ depends only on $M_{t m}$. This dependence is shown in Fig. 12, from which it is clear that rather high values of $M_{t p}$ are required when $T_{p} / T_{c}$ is small. This is due simply to the very rapid decrease of $T_{p}{ }^{*}$ with increasing $r^{*}$ for these cases; however, it is important to note that the mass flow capacity of the vortex depends on $M_{t m}$ [Eq. (8)], and that as $T_{p} / T_{c}$ (or $T_{p}{ }^{*}$ ) decreases, it will become more difficult to obtain satisfactorily high values of $M_{l m}$.

From Eq. (8), the mass flow capacity of the vortex per unit length, $\mathfrak{T}_{1}$, is related to $M_{t m}$ and the diffusion coefficient. Using Eq. (16), the relation may be written as

$$
\begin{aligned}
\mathfrak{K}_{1}=\frac{3\left[2 \pi k m_{1}\left(1+m_{1} / m_{2}\right)\right]^{1 / 2}}{8 d_{12}{ }^{2}} & \left(\frac{m_{2}}{m_{1}}-1\right) \times \\
& \frac{\gamma T_{p}^{1 / 2}}{\left[1-\left(w_{c} / w_{m}\right)\right]} \Psi
\end{aligned}
$$

where $\Psi=M_{t m}{ }^{2} / T_{p}{ }^{* 1 / 2}$. Since $T_{p}{ }^{*}$ is independent of $w_{m}$, so is $\Psi$; its dependence on $M_{t m}$ and $T_{p} / T_{c}$ is shown in Fig. 13. It is clear that for given values of $T_{p}$ and $M_{t m}, \mathfrak{T}_{1}$ increases with increasing $T_{c} / T_{p}$; however, if $M_{t p}$ is held fixed, $\Psi$ decreases with increasing $T_{d} / T_{p}$, as indicated by the lines of constant $M_{t p}$ included in Fig. 13.

The radius $r_{c}$ of the inner boundary of the vortex effectively determines the maximum possible length-todiameter ratio of a vortex such as that in Fig. 1, since

TABLE 2

\begin{tabular}{ccc}
\hline \multicolumn{3}{c}{ Maximum of $T_{d} / T_{p}$ for Subcritical Solutions } \\
\hline$M_{t m}$ & $w_{m} 0.2-1.0$ & $w_{m}=4.0$ \\
\hline 0.5 & 4.2 & 3.8 \\
0.7 & 3.0 & 2.5 \\
1.0 & 2.9 & 2.4 \\
\hline
\end{tabular}


all of the mass flow must leave the vortex axially near its center, and the radius of the exit nozzle must be less than $r_{c}$. Thus, the ratio $r_{c} / r_{p}$ is an important performance parameter. Its dependence on $M_{t m}, w_{m}$, and $T_{c} / T_{p}$ is shown in Fig. 14. In contrast to $M_{t p}$ and $\Psi, r_{c} / r_{p}$ is dependent to some extent on $w_{m}$. This is because the profile of $w$ depends on the magnitude of $w$ itself, and not $w / w_{m}$ alone, near $r_{c}$, where $w \approx w_{c}$. All the values or $r_{c} / r_{p}$ are near 0.5 or larger, so that for the range of $M_{t m}$ covered in Fig. 14, the heavy gas is essentially confined to the outer half of the vortex radius.

It is convenient to refer the heating parameter to the conditions in the vortex at its periphery. Thus,

$$
g_{p}=-\frac{2 \pi Q \sigma_{f} \phi n_{2 p} r_{p}^{2}}{\mathfrak{M}_{1}\left(1+w_{c}\right) C_{p 1} T_{p}}=g_{m}\left(n_{2 p}{ }^{*} r_{p}{ }^{*} / T_{p}{ }^{*}\right)
$$

is a direct measure of the rate of enthalpy increase in the outer portion of the vortex, and if it is given as a function of $T_{c} / T_{p}, v_{m}$, and $M_{t m}$, it permits the calculation of the neutron flux, $\phi$, required to give any specified temperature ratio. The dependence of $g_{p}$ on $w_{m}$ and $T_{c} / T_{p}$ for $M_{t m}=0.7$ is shown in Fig. 15. It increases rapidly as $T_{c} / T_{p}$ increases, as would be expected. The large difference between the supercritical and subcritical cases for a given $T_{c} / T_{p}$ is due simply to the fact that $w_{p} / w_{m}=1$ for the former and $w_{p} / w_{m}=$ 0.1 for the latter. If the values of $g_{p}$ for the subcritical case are multiplied by 10 , they very nearly fall on the curve for the supercritical case, in the range of $T_{c} / T_{p}$ where the two overlap.

Finally, to complete the description of the vortex from the nuclear standpoint, we need some measure of the total amount of fissionable gas and of propellant gas in it. We may define a mean propellant density by

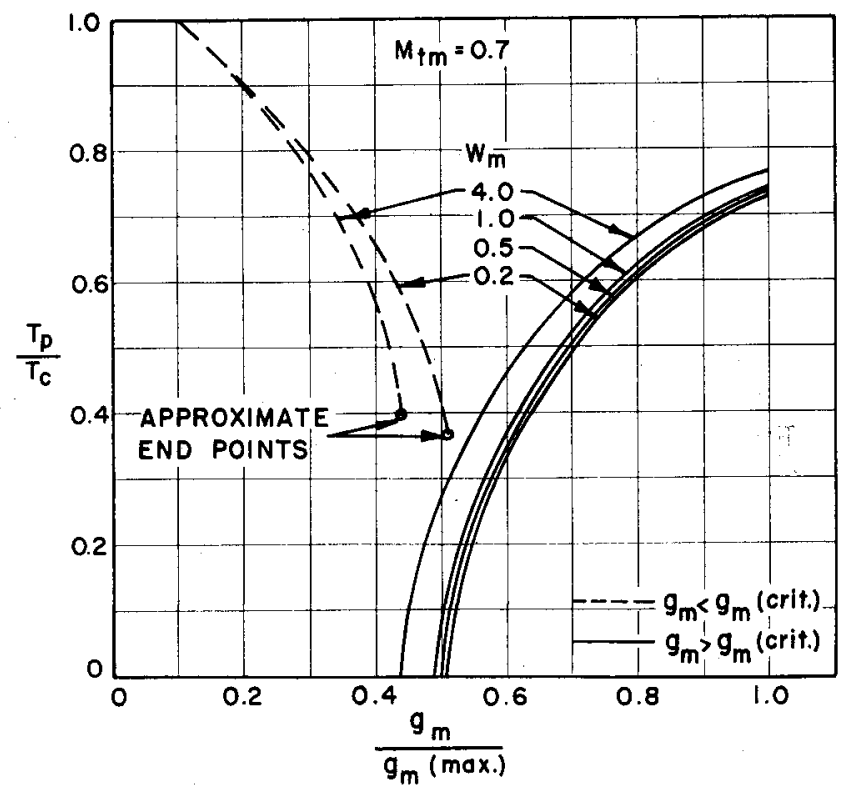

Fig. 10. Dependence of the overall temperature ratio, $T_{p} / T_{c}$ on the ratio of the heating parameter $g_{m}$ to its maximum permissible value; for $M_{t m}=0.7$.

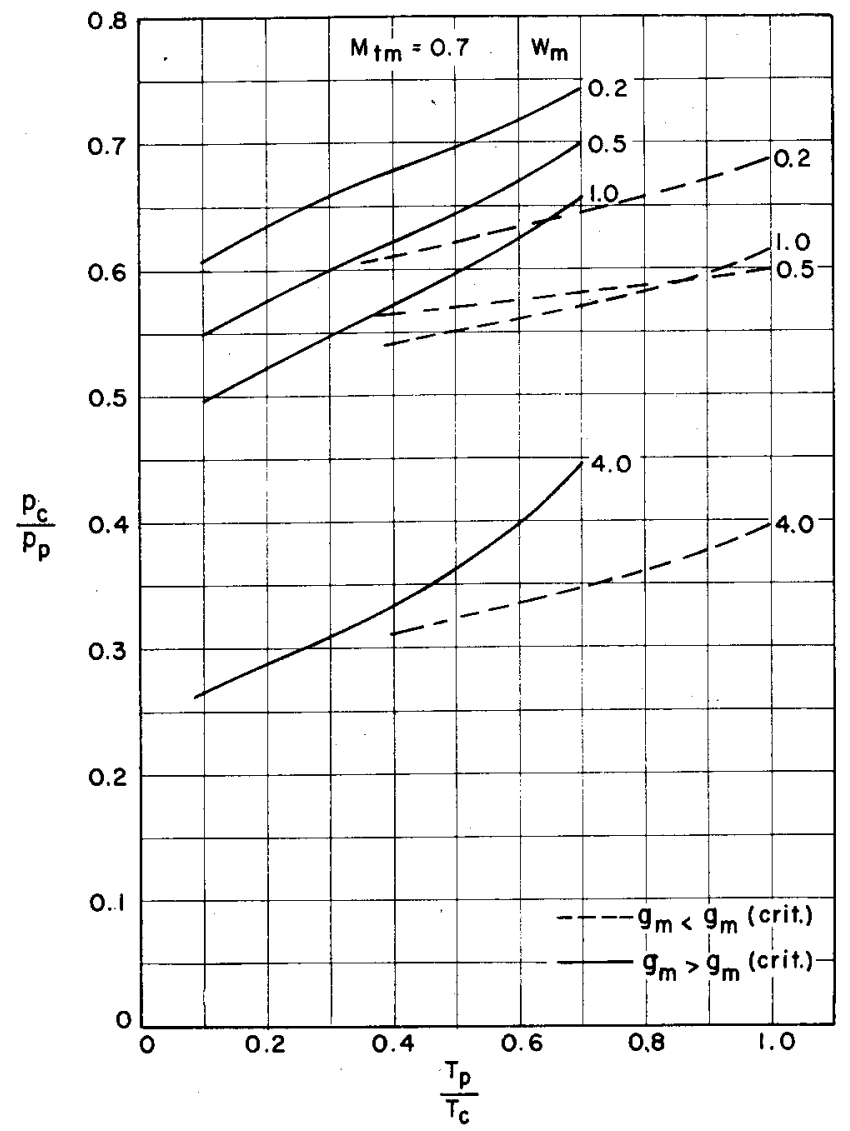

Frg. 11. Dependence of the overall pressure ratio, $p_{c} / p_{p}$, on the overall temperature ratio, $T_{p} / T_{c} ;$ for $M_{\iota m}=0.7$.

$$
\frac{\bar{\rho}_{1}}{\rho_{1 p}}=\frac{\rho_{1 c}{ }^{*}{ }^{*}{ }_{c}{ }^{* 2}+2 \int_{r_{c}^{*}}^{\gamma_{p}{ }^{*}} \rho_{1}{ }^{*} r^{*} d r^{*}}{\rho_{1 p}{ }^{*} r_{p}{ }^{* 2}}
$$

if we assume $\rho_{1}^{*}$ is constant at $\rho_{1 c}{ }^{*}$ for $r^{*}<r_{c}^{*}$. Similarly, if we define a mean density ratio, $\bar{w}$, by

$$
\frac{\bar{w}}{w_{m}}=2 \frac{\rho_{1 p}}{\bar{\rho}_{1}} \frac{T_{p}^{*}}{p_{p}^{*} r_{p}^{* 2}} \int_{r_{c}^{*}}^{r_{p}^{*}} n_{2}{ }^{*} r^{*} d r^{*}
$$

then the average density of the fissionable gas is $\rho_{2}{ }^{*}=$ $\vec{w}\left(\bar{\rho}_{1} / \rho_{1 p}\right) \rho_{1 p} . \quad \bar{\rho}_{1} / \rho_{1 p}$ and $\vec{w} / w_{m}$ are given in Figs. 16 and 17 for $M_{t_{m}}=0.7$. We see that $\bar{w} / \bar{w}_{m}$ is largest for the supercritical cases, because $w=w_{m}$ both at $r^{*}=1$ and at $r^{*}=r_{p}{ }^{*}$; and that it has a maximum, becatse $w$ deviates more from $w_{m}$ in the outer portions of the vortex as $T_{p} / T_{c}$ decreases. $\bar{\sigma}_{1} / \rho_{1 p}$ increases monotonically with $T_{p} / T_{c}$, simply because of the influence of the temperature on the density. There is also some influence of $w_{m}$, which arises from the variation of the pressure drop with $w_{m}$

\section{Performance and Criticality Estimates}

The significance of the results obtained from the preceding analysis of the heating separation process may perhaps be better revealed if incorporated into the performance analysis of a gaseous vortex reactor for rocket propulsion. In the present section the characteristic performance parameters of the rocket are 


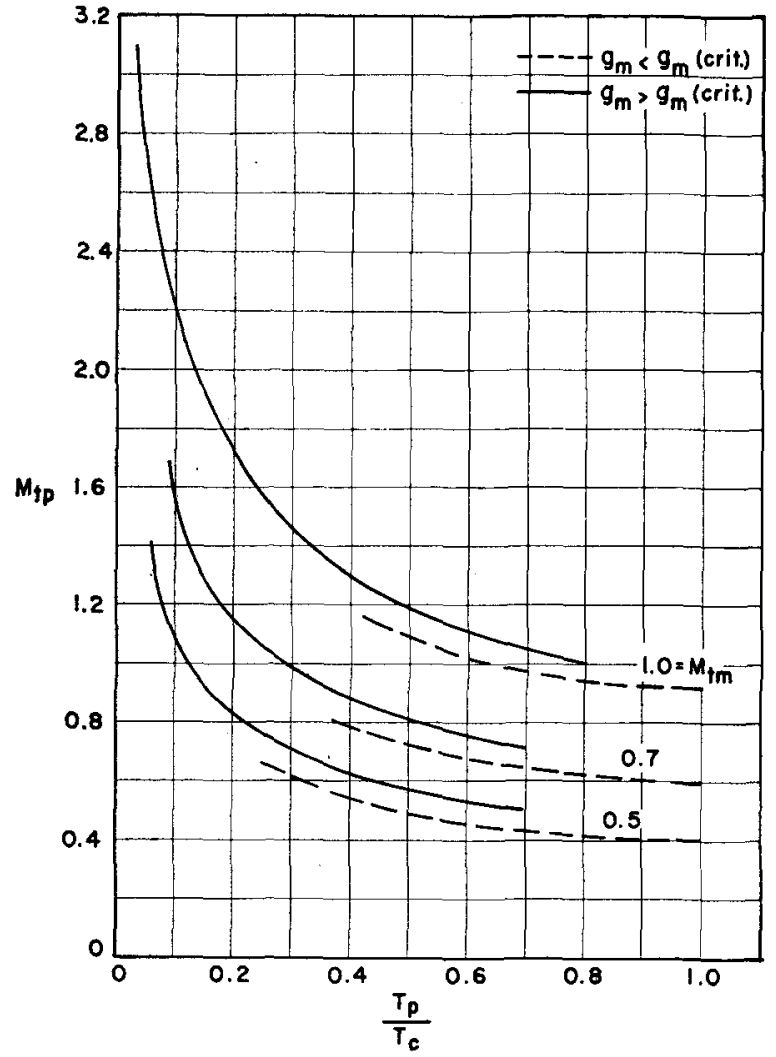

Fig. 12. Dependence of the tangential Mach number at the periphery, $M_{t p}$ on the overall temperature ratio, $T_{p} / T_{c}$.

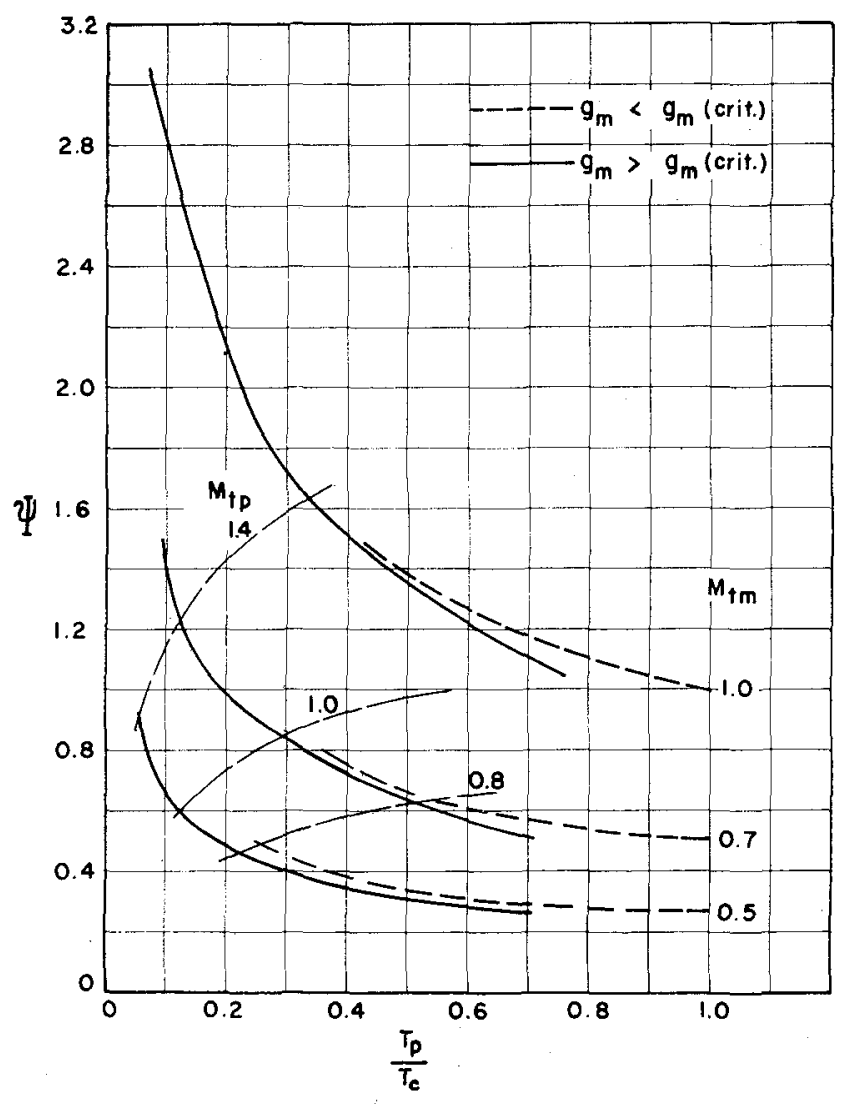

Frg. 13. Dependence of the relative mass flow capacity, $\Psi$, on the overall temperature ratio, $T_{p} / T_{c}$. calculated in terms of the parameters derived from the separation analysis and from the nuclear configuration. To be definitive, such an analysis would have to include an optimization of the entire system for some mission, and this would imply a detailed study of many aspects of the vehicle configuration, as well as those of the power plant. The intent of the present analysis, however, is much more modest; it is simply to provide a physical feeling for the several parameters which characterize the performance of the vortex tube as applied to rocket propulsion.

To this end, the requirements imposed on the reactor system by the overall energy balance, and by criticality, will be estimated. The weight, thrust-to-weight ratio, neutron flux, and various other parameters will then be given for some representative examples.

It is assumed in these computations that the reactor core consists of a cylindrical bundle of vortex tubes such as that shown in Fig. 1, the diameter being equal to the length $l$ of an individual tube. Both graphite and beryllium oxide will be considered as moderators. In those cases where the temperature ratio $T_{c} / T_{p}$ is such that heat must be added to the propellant, by fissionable material, before it enters the vortex tubes, it will be assumed that the fissionable material not contained in the tubes is uniformly dispersed in the moderator. Some reduction in overall size and weight should be obtainable by concentrating this solid fuel in as small a volume as possible, but this refinement will not be considered here.

\section{Energy Balance}

Although most of the fission power generated in the vortex tubes appears directly as an increase in the enthalpy of the gas mixture, there will always be some fraction $\zeta$ carried by radiation which penetrates the gas and is deposited directly in the surrounding solid regions of the reactor. The radiation mechanism is both thermal and nuclear (gamma rays and neutrons); therefore, in general $\zeta$ will depend on the properties of the gas and the nuclear characteristics of the reactor.

In the special case that the propellant gas is the only available heat sink, the propellant must be passed first through the solid regions of the engine to remove the energy deposition represented by $\zeta$, and thence into the vortex tubes for further heating. Consider now a system in which some of the fissionable material is contained in solid fuel plates so that a fraction $f$ of the fission reactions occur in the solid regions. If $P$ is the total power generated in this system, $h_{c}$ the enthalpy per unit mass of propellant corresponding to the temperature in the vortex core, and $\dot{m}$ the mass flow rate of propellant, then $P=\dot{m} h_{c}$. The total power deposited in the solid regions $P_{s}$ is then

$$
P_{s}=f P+\zeta P_{c}=[f+\zeta(1-f)] P
$$

where $P_{c}=(1-f) P$, the fission power released directly into the gas mixture in the cavity. Further, if $h_{p}$ is the enthalpy corresponding to the maximum tempera- 
ture in the solid, then for the present case $P_{s}=\dot{m} h_{p}$, and we obtain

$$
h_{c} / h_{p}=1 /[f+\zeta(1-f)]
$$

Now, the specific impulse $I$ for a rocket engine with a completely expanded nozzle is simply $g^{2} I^{2} / 2=h$, where $g$ is the gravitational constant. Thus, the ratio given in Eq. (30) is the square root of the corresponding specific impulses. The significance of this result may be appreciated if one thinks of the quantity $I_{p}$ as the maximum possible specific impulse attainable in a solid-fuel (temperature-limited) reactor. Then $I_{c} / I_{p}$ would be the performance gain to be realized in incorporating some gas-phase fission heating. Fig. 18 shows this ratio as a function of $f$ for the case $\zeta=0.1$. Evidently, with an all gas-phase reactor $(f=0)$, it is possible to achieve specific impulses about three times as large as with an all solid-fuel nuclear engine.

If the restriction that all heat must be transferred to the propellant is removed by providing radiators for rejection of heat from the solid parts of the reactor, $h_{c} / h_{p}$ can, at least in principle, be larger than the value given by Eq. (30). However, if an appreciable fraction of the total power is radiated, the radiator weight will be so large that the thrust-to-weight ratio will necessarily be much less than unity. This is not necessarily a disadvantage for space propulsion applications.

\section{Criticality}

Estimates of the critical size of the reactor have been obtained from two-group, two-region calculations for a completely reflected cylinder. The calculations were done on a digital computer using a two-group, two-region code, and a reflector savings program.*

The reactor core was taken to be a homogeneous mixture of moderator, plutonium and hydrogen. The proportions of the latter two were determined from the separation analysis. Fast fission was neglected and the resonance escape probability was taken as unity.

The thickness of the beryllium reflector for each reactor and the void fraction $\beta$ of the core were selected to minimize the combined core and reflector weight. The calculations made by Lafyatis and Nelson indicated that $\beta=0.6$ would be near optimum for most configurations of interest.

The fission cross section of plutonium has a marked resonance at a neutron energy of 0.3 electron volts, which coincides with the mean thermal neutron energy (Maxwell distribution) for a temperature of $2,330^{\circ} \mathrm{K}$. Thus, the neutron temperature has an important effect on the critical mass for the systems considered here. It was assumed that the neutron temperature was equal to the moderator temperature, which in turn was taken equal to $T_{p}$.

\section{Rocket Engine Performance}

For the present purpose, the performance of the vor-

* The calculations were made by P. G. Lafyatis and M. L. Nelson of the Oak Ridge National Laboratory, USAEC.

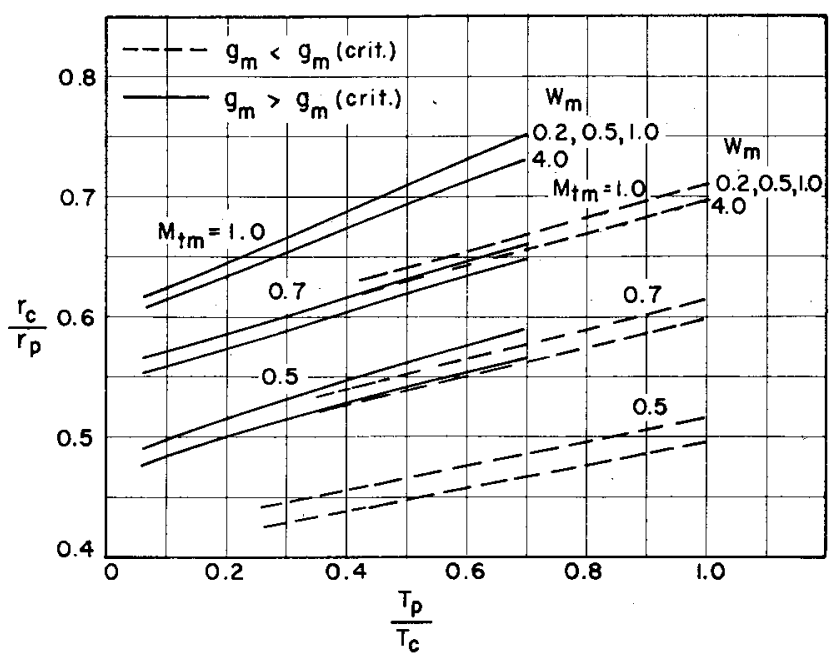

FIG. 14. Dependence of the ratio of exit radius to tube radius on the overall temperature ratio, $T_{p} / T_{c}$.

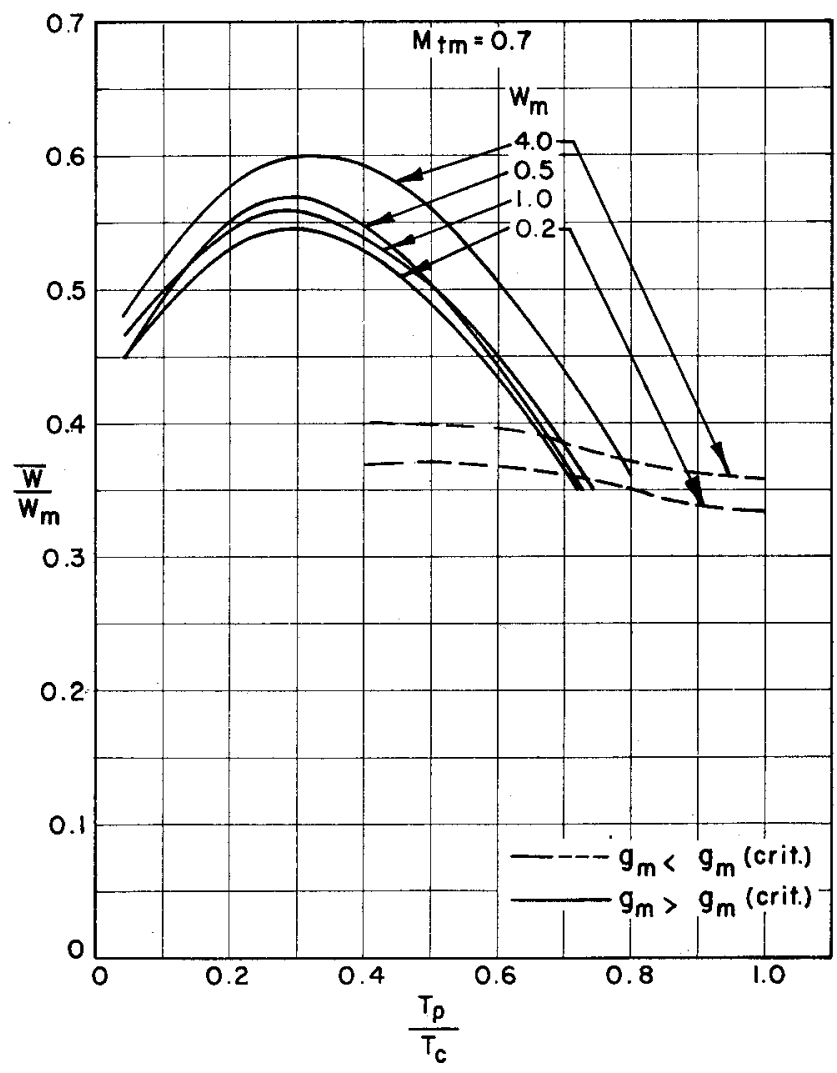

Fig. 15. Dependence of the ratio of average to maximum density on the overall temperature ratio, $T_{p} / T_{c} ;$ for $M_{t m}=0.7$.

tex tube reactor may be characterized by the specific impulse, the total thrust, and the ratio of thrust to reactor (core plus reflector) weight.

The specific impulse is determined from the total energy content $H_{c}$ of the propellant gas at the vortex "core" condition. In the present system, the total energy is given by the enthalpy per unit mass of gas, $h_{c}$, plus the kinetic energy due to the tangential velocity $v_{c}$ of the propellant at the core radius $r_{c}$. Thus, 


$$
H_{c}=h_{c}+\left(v_{c}^{2} / 2\right)
$$

If we use the fact that the tangential velocity in the vortex is inversely proportional to the radius, then we can relate $v_{c}$ to $v_{p}$, the tangential velocity at the tube periphery, thus:

$$
v_{c}^{2}=\gamma_{c} R_{c} T_{p} M_{t p}^{2}\left(r_{p} / r_{c}\right)^{2}
$$

where $\gamma_{c}, R_{c}$ refer to the core conditions; and Eq. (31) may be written

$$
H_{c}=h_{c}\left[1+\frac{\gamma-1}{2} M_{t p}{ }^{2}\left(\frac{r_{p}}{r_{c}}\right)^{2}\left(\frac{T_{p}}{T_{c}}\right)\right]
$$

where we have used $h_{c}=C_{p c} T_{c}$. For the systems to be considered here, the second term in Eq. (32) is in the order of 0.2 and will be neglected in these computations. This means that we are underestimating the energy available to the gas and will therefore obtain a slightly conservative estimate of engine performance.

The total thrust $F$ from the assembly of vortex tubes in the reactor is

$$
F=N l \Re_{1} c
$$

where $l$ is the length of the tubes, $N$ the number of tubes, $\mathfrak{T}_{1}$ is the mass flow rate per unit length of tube, and $c$ is the rocket exhaust velocity, where $c^{2} / 2=h_{c}$. Now, the product $\mathfrak{M}_{1} l$ is related to the nozzle throat radius $r_{t}$ by

$$
\mathfrak{M}_{1} l=\pi r_{t}^{2} \rho_{i} a_{t}
$$

where $\rho_{t}$ is the propellant gas density and $a_{t}$ the velocity of sound at the throat. These are related to the core conditions by

$$
\rho_{t}=\rho_{1 c}\left(\frac{2}{\gamma+1}\right)^{1 /(\gamma-1)}
$$

and

$$
a_{t}^{2}=2\left(\frac{\gamma-1}{\gamma+1}\right) h_{c}
$$

Thus, Eq. (34) may be written

$$
\begin{aligned}
\mathfrak{T t}_{1} l=\pi r_{p}^{2} \rho_{1 c}\left[(\gamma-1) h_{c}\right]^{1 / 2} \times \\
\\
\left(\frac{2}{\gamma+1}\right)^{(\gamma+1) / 2(\gamma-1)}\left(\frac{r_{c}}{r_{p}}\right)^{2}\left(\frac{r_{t}}{r_{c}}\right)^{2}
\end{aligned}
$$

Substitution into the thrust equation yields

$$
\begin{aligned}
F=(\pi / 4) \beta l^{2} \gamma p_{c} & \left(\frac{2}{\gamma-1}\right)^{1 / 2} \times \\
& \left(\frac{2}{\gamma+1}\right)^{(\gamma+1) / 2(\gamma-1)}\left(\frac{r_{c}}{r_{p}}\right)^{2}\left(\frac{r_{t}}{r_{c}}\right)^{2}
\end{aligned}
$$

where we have used the relation $\rho_{1 c}=\gamma p_{c} /(\gamma-1) h_{c}$ and introduced the expression for the reactor core volume $\pi r_{p}^{2} N l / \beta$, which is $\pi l^{3} / 4$ for a square cylinder, to eliminate $N$. The quantity $r_{t} / r_{c}$ is the ratio of nozzle

\begin{tabular}{|c|c|c|c|c|c|c|}
\hline \multicolumn{7}{|c|}{ Numerical Examples of Vortex Tube Reactors* } \\
\hline \multirow[b]{2}{*}{ Parameter } & \multicolumn{6}{|c|}{ Case Number } \\
\hline & 1 & 2 & 3 & 4 & 5 & 6 \\
\hline$T_{c} / T_{p}$ & 1.56 & 1.56 & 1.56 & 4.05 & 4.05 & 4.05 \\
\hline$w_{m}$ & 0.5 & 1.0 & 4.0 & 0.5 & 1.0 & 4.0 \\
\hline$M_{t p}$ & 1.0 & 1.0 & 1.0 & 1.0 & 1.0 & 1.0 \\
\hline$T_{c},{ }^{\circ} \mathbf{R}$ & 7,020 & 7,020 & 7,020 & 10,000 & 10,000 & 10,000 \\
\hline$T_{p},{ }^{\circ} \mathrm{R}$ & 4,500 & 4,500 & 4,500 & 2,470 & 2,470 & 2,470 \\
\hline$p_{e}$, atm. & 100 & 100 & 100 & 100 & 100 & 100 \\
\hline$p_{p}$, atm. & 213 & 231 & 498 & 171 & 188 & 338 \\
\hline$r_{c} / r_{p}$ & 0.66 & 0.66 & 0.65 & 0.59 & 0.59 & 0.58 \\
\hline$\tilde{w} / \omega_{m}$ & 0.34 & 0.35 & 0.36 & 0.56 & 0.56 & 0.54 \\
\hline & 0.96 & 0.96 & 1.50 & 56 & 56 & 81 \\
\hline $\mathfrak{S T}_{1}$, lb./sec. ft. & 0.0198 & 0.0198 & 0.0198 & 0.0121 & 0.0121 & 0.0121 \\
\hline$I$, sec. & 1,196 & 1,196 & 1,196 & 1,426 & 1,426 & 1,426 \\
\hline$\eta_{2 g}, \mathrm{~cm}^{3}$ & $0.40 \times 10^{18}$ & $0.85 \times 10^{18}$ & $5.2 \times 10^{18}$ & $0.49 \times 10^{18}$ & $1.01 \times 10^{18}$ & $5.12 \times 10^{18}$ \\
\hline$l, \mathrm{ft}$. & $\begin{array}{l}15.4 \\
(14.0)\end{array}$ & $\begin{array}{l}10.2 \\
(8.5)\end{array}$ & $\begin{array}{l}3.8 \\
(4.1)\end{array}$ & $\begin{array}{l}16.8 \\
(16.7)\end{array}$ & $\begin{array}{l}11.7 \\
(10.1)\end{array}$ & $\begin{array}{l}4.5 \\
(4.5)\end{array}$ \\
\hline$F /\left(r_{t} / r_{c}\right)^{2}, 1 \mathrm{~b}$ & $\begin{array}{l}15.3 \times 10^{6} \\
\left(12.6 \times 10^{6}\right)\end{array}$ & $6.7 \times 10^{6}$ & $\begin{array}{l}0.90 \times 10^{6} \\
\left(102 \times 10^{6}\right.\end{array}$ & $14.6 \times 10^{6}$ & $7.02 \times 10^{6}$ & $1.01 \times 10^{6}$ \\
\hline Weight, lb. & 180,000 & 75,000 & 10,000 & 230,000 & 100,000 & 15,000 \\
\hline & $(120,000)$ & $(35,000)$ & $(5,300)$ & $(180,000)$ & $(55,000)$ & $(6,700)$ \\
\hline$\left[F /\left(r_{t} / r_{c}\right)^{2}\right] /$ weight & $\begin{array}{l}85 \\
(98)\end{array}$ & 89 & 90 & 64 & 70 & 67 \\
\hline$N /\left(r_{i} / r_{c}\right)^{2}$ & 55,400 & $\begin{array}{l}(136) \\
36,700\end{array}$ & 13,300 & 66,200 & $\begin{array}{l}(94) \\
46,100\end{array}$ & $(150)$ \\
\hline & $(50,400)$ & $(30,600)$ & $(14,300)$ & $(65,800)$ & $(39,800)$ & $(17,100)$ \\
\hline$\phi /\left(r_{t} / r_{c}\right)^{2}$, & & & & & & \\
\hline neutrons/cm. ${ }^{2}$-sec. & $\begin{array}{l}0.78 \times 10^{18} \\
\left(0.85 \times 10^{18}\right)\end{array}$ & $\begin{array}{l}0.53 \times 10^{18} \\
\left(0.64 \times 10^{18}\right)\end{array}$ & $\begin{array}{l}0.26 \times 10^{18} \\
\left(0.24 \times 10^{18}\right)\end{array}$ & $\begin{array}{l}1.6 \times 10^{18} \\
\left(1.6 \times 10^{18}\right)\end{array}$ & $\begin{array}{l}1.0 \times 10^{18} \\
\left(1.2 \times 10^{18}\right)\end{array}$ & $\begin{array}{l}0.54 \times 10^{18} \\
\left(0.54 \times 10^{18}\right)\end{array}$ \\
\hline$r_{p}\left(r_{t} / r_{c}\right)$, in & $\begin{array}{l}0.305 \\
(0.291)\end{array}$ & $\begin{array}{l}0.248 \\
(0.226)\end{array}$ & $\begin{array}{l}0.154 \\
(0.159)\end{array}$ & $\begin{array}{l}0.304 \\
(0.303)\end{array}$ & $\begin{array}{l}0.253 \\
(0.236)\end{array}$ & $\begin{array}{l}0.160 \\
(0.160)\end{array}$ \\
\hline
\end{tabular}
throat radius to the radius at which $w \rightarrow w_{c}$ in the vortex tube. Note that $0<r_{t} / r_{c}<1$. It can be seen from Eq. (36) that the thrust of this system is actually limited by the size of the area which can be provided at the back face of the reactor for the exhaust nozzles, without allowing the fissionable material to be blown out. This area is $\pi \beta l^{2}\left(r_{c} / r_{p}\right)^{2} / 4$. The thrust is also proportional to the pressure $p_{c}$, but independent of the "core" temperature $T_{c}$. The ratio $r_{c} / r_{p}$ is of course

Table 3

${ }^{*}$ Performance parameters based on $\beta=0.6, \gamma=1.31, Q=7.36 \times 10^{-12}$ cal. $/$ fission, $C_{p 1}=3.54$ cal. $/ \mathrm{gm} .{ }^{\circ} \mathrm{K} .$, and $\sigma_{f}=975$ barns for $T_{p}=2,470^{\circ} \mathrm{R}$. , and $C_{p 1}=5.25 \mathrm{cal} . / \mathrm{gm} .{ }^{\circ} \mathrm{K}$. and $\sigma_{f}=810$ barns for $T_{p}=4,500^{\circ} \mathrm{R}$. 
determined by the diffusion process in the tubes. It depends principally upon $M_{t m}$, increasing as $M_{t m}$ increases.

Eq. (36) contains the factor $\left(r_{t} / r_{c}\right)^{2}$, which may have any value from zero to unity. Thus, for a given set of vortex tube parameters and critical reactor size, the thrust may have any value from zero to that given by Eq. (36) for $r_{t} / r_{c}=1$. As this ratio increases, the number of tubes in the reactor increases, each tube decreasing in diameter. This may be seen by equating Eqs. (33) and (36) and solving for $N$. Then, using $h_{c} \approx C_{p c} T_{e}$, we obtain

$N=\frac{\pi \beta l p_{c} \gamma^{1 / 2}}{4 \mathbb{M}_{1}\left(R T_{c}\right)^{1 / 2}}\left(\frac{2}{\gamma+1}\right)^{\frac{\gamma+1}{2(\gamma-1)}}\left(\frac{r_{c}}{r_{p}}\right)^{2}\left(\frac{r_{t}}{r_{c}}\right)^{2}$

For a given set of vortex tube parameters, $\mathfrak{N}_{1}$ and $r_{c} / r_{p}$ are fixed. Then, for fixed $p_{c}$ and $T_{c}$, the number of tubes is proportional to $\left(r_{t} / r_{c}\right)^{2}$.

The average neutron flux required to give the heat release rates implied by these performance parameters may be determined from Eq. (27), which gives

$$
\phi=g_{p} \frac{\left(1+w_{c}\right) C_{p 1} T_{p}}{2 n_{2 p} \sigma_{f} Q}\left(\frac{\Re_{1}}{\pi r_{p}^{2}}\right)
$$

where $h_{p}=C_{p 1} T_{p}$. The last factor above may be eliminated by applying Eq. (35). If we note also that

$$
n_{2 p}=w_{p} \rho_{1 p} / m_{2}=w_{p}\left(p_{c} / m_{2} R T_{c}\right)\left(p_{p} / p_{c}\right)\left(T_{c} / T_{p}\right)
$$

then the expression for $\phi$ may be written

$$
\begin{aligned}
\phi= & \frac{g_{p}\left(1+w_{c}\right) m_{2} C_{p 1} T_{c}\left(\gamma R T_{c}\right)^{1 / 2}}{2 w_{p} l \sigma_{f} Q} \times \\
& \left(\frac{T_{p}}{T_{c}}\right)^{2}\left(\frac{2}{\gamma+1}\right)^{\frac{\gamma+1}{2(\gamma-1)}}\left(\frac{p_{c}}{p_{p}}\right)\left(\frac{r_{c}}{r_{p}}\right)^{2}\left(\frac{r_{c}}{r_{c}}\right)^{2}
\end{aligned}
$$

$g_{p}$ increases with increasing $T_{c} / T_{p}$ (Fig. 15), and $C_{p 1}$ also increases with increasing $T_{c}$, so that $\phi$ will increase quite rapidly with increasing $T_{c}$.

\section{Numerical Examples}

It was mentioned previously that the enthalpy ratio $h_{c} / h_{p}$ (or the temperature ratio $T_{c} / T_{p}$ ) is the most important parameter in determining the characteristics of the vortex tube. On the other hand, the fissionable material concentration determines the overall size and weight of the reactor. Accordingly, examples have been selected with two representative values of $T_{c} / T_{p}$, and three values of $w_{m}$. The pressure at the vortex core has been taken as 100 atmospheres for all cases, so that $w_{m}$ is nearly a direct measure of the fuel concentration in the gas phase.

Cases 1,2 , and 3 have $T_{c} / T_{p}=1.56$. This temperature ratio corresponds to about half the heat being added to the propellant by solid fuel elements, and half by the vortex tubes. The temperature leaving the solid reactor, $T_{p}$, has been set at the upper limit for graphite fuel elements, namely, $4,500^{\circ} \mathrm{R}$; hence the chamber temperature, $T_{c}$, is $7,020^{\circ} \mathrm{R}$. This is of the same order as the chamber temperature for contem-

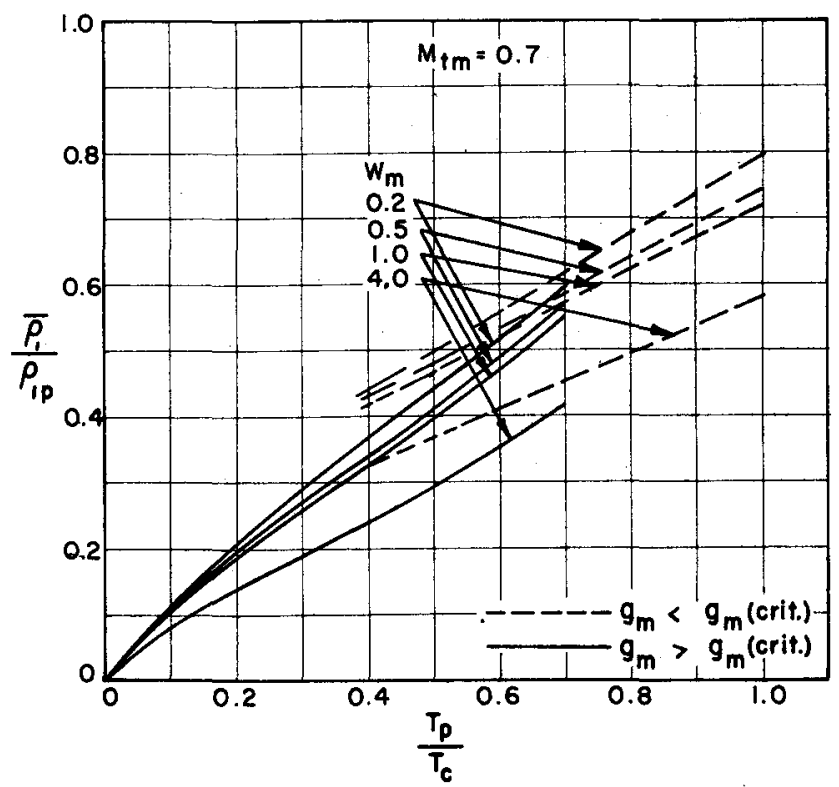

Fig. 16. Dependence of the ratio of average to peripheral propellant densities on the overall temperature ratio, $T_{p} / T_{c}$; for $M_{t m}$ $=0.7$.

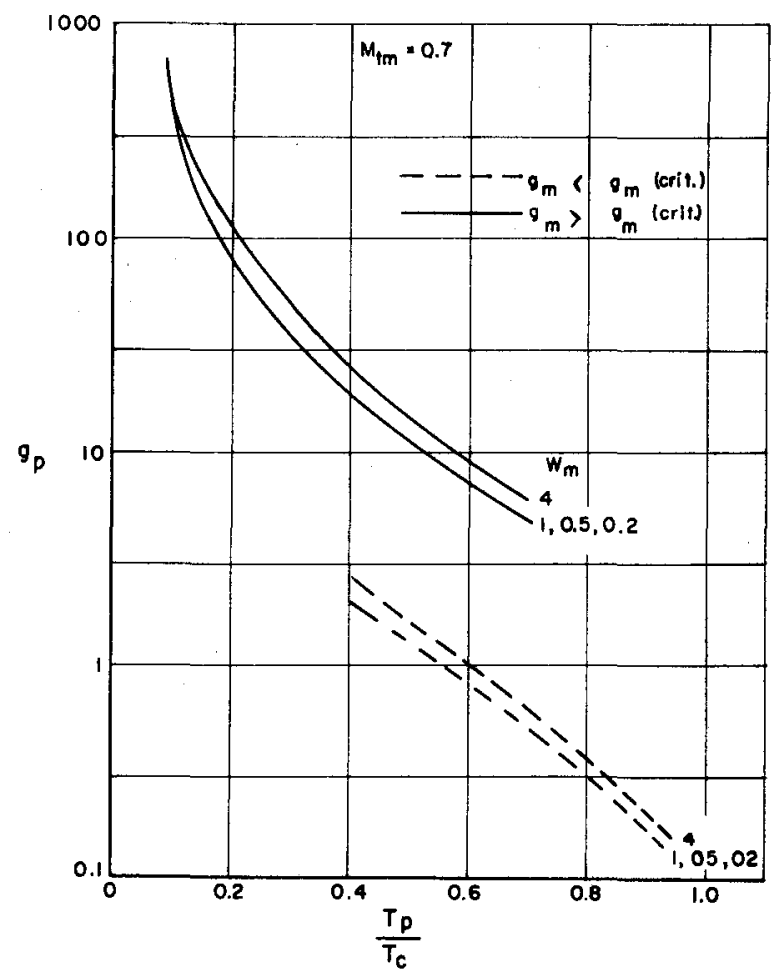

FIG. 17. Dependence of the heating parameter, $g_{p}$, on the overall temperature ratio, $T_{p} / T_{c} ;$ for $M_{t m}=0.7$.

porary chemical rocket motors-e.g., liquid $\mathrm{O}_{2}$ and $\mathrm{JP}-4$ give $T_{c} \approx 6,300^{\circ} \mathrm{R}$.

Cases 4,5 , and 6 have the largest values of $T_{c} / T_{p}$ possible for $T_{c}=10,000^{\circ} \mathrm{R}$. They represent systems with chamber temperatures considerably higher than the best obtainable with chemical rockets. At the same time, the temperatures entering the vortex tubes are considerably below the limits for graphite or the refrac- 


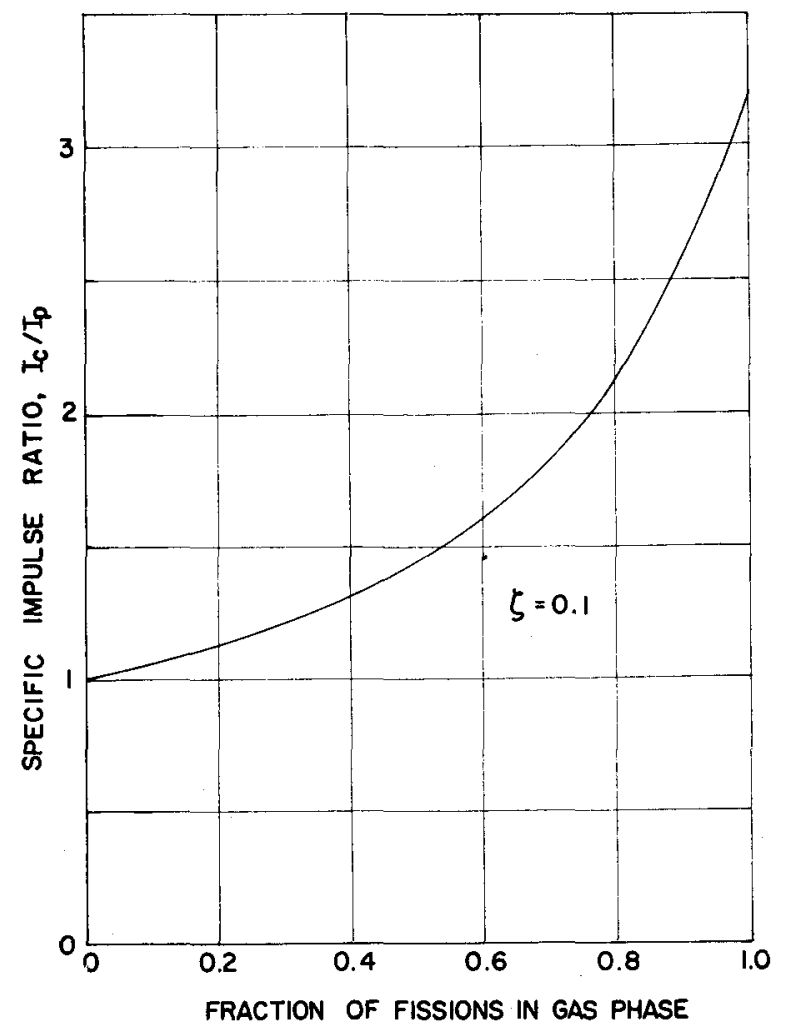

Fig. 18. Specific impulse ratio, $I_{e} / I_{p}$, as function of gas-phase fission heating.

tory metals. These systems have all fissionable material in the gas phase.

Some characteristics of these reactors are shown in Table 3. The values for beryllium-oxide-moderated reactors are shown in parentheses. The first few quantities listed are characteristic of the vortex tube itself, and hence are independent of the moderator used.

Although the reactor weights (core plus reflector) are very high for fuel concentrations of the order of $0.5 \times 10^{18} \mathrm{~cm}^{-3}$, they are quite reasonable if the concentration can be increased to about $5.0 \times 10^{18} \mathrm{~cm}^{-3}$ These higher fuel concentrations imply high pumping pressures, however. For Case $3, p_{p}$ is 498 atmospheres, or $7,320 \mathrm{psi}$. The pumping pressure must be taken as about twice this figure, to allow for the pressure drops in the inlet nozzles and solid reactor. It should be noted that the increase in average fuel concentration, $\bar{n}_{2}$, by a factor of 10 from Case 1 to Case 3 requires only a little more than doubling of the pumping pressure. This increase is due to the higher pressure drop caused by increasing $w_{m}$. To achieve the same concentration increase by raising the general pressure level with constant $w_{m}$, it would be necessary to increase the pumping pressure tenfold, to about 4,000 atmospheres. The beryllium-oxide-moderated reactors are in all cases lighter than the corresponding graphite-moderated reactors. This is due to the decreased neutron-slowingdown length and decreased diffusion length in beryllium oxide as compared to graphite.

Although the reactor weights are rather high, the thrust-to-weight ratios are also quite high if $r_{t} / r_{c}$ is near unity. It may, however, be more realistic to take $r_{t} / r_{c}$ a little less than unity, to allow for threedimensional flow effects in the long, thin, vortex tubes. If $r_{t} / r_{c}$ is taken as 0.5 , for example, the beryllium-oxidemoderated reactor described in Case 3 has a thrust of $255,000 \mathrm{lbs}$., and a thrust-to-weight ratio of 48 . The latter figure is somewhat lower than that for a chemical system; however, the specific impulse is at least twice that of the best chemical rockets. In order to give this performance the reactor would contain 950 vortex tubes, each 0.32 in. in diameter. The average neutron flux would be $5.2 \times 10^{16}$ neutrons/sec.-cm. ${ }^{2}$

It seems from comparison of Cases 3 and 6 , for example, that as $T_{c}$ is increased, the critical size and weight of the reactor increase. This effect is due to a lower average fuel concentration in the higher temperature reactor. The gas-phase fuel concentrations, $\bar{n}_{2 g}$, are about the same in the two cases, but the lowertemperature reactor contains considerable solid fuel.

\section{Concluding Remarks}

In conclusion, it seems worth while to summarize the more important results of the analysis. One of these is that the mass flow capacity per unit length of the vortex tube, as limited by the rate of diffusion of propellant through the fissionable gas, is independent of the tube diameter. Thus, even though the mass flow per unit of tube length is only of the order of 0.01 pounds per second per foot, the mass flow capacity per unit volume of an assembly of small-diameter tubes can be quite large.

A second important conclusion is that containment of the fissionable material within the vortex, away from its outer boundary, is possible only if the pressure gradient decreases monotonically with increasing radius. As a corollary of this, there is a critical heating rate above which such containment is impossible.

Insofar as the vortex containment mechanism limits the performance of the gaseous nuclear rocket, the performance capability is very high. It may well be that the performance will actually be limited by other factors, such as radiant heat transfer from the gas to solids, the difficulty of maintaining fissionable material in gaseous form at the required concentrations and temperatures, and the difficulty of generating vortices with the required low radial mass-flow rates and high tangential velocities. The potential of the gaseous fission rocket seems to warrant intensive study of these problems.

\section{References}

\footnotetext{
1 Kerrebrock, J. L., and Meghreblian, R. V., An Analysis of Vortex Tubes for Combined Gas-Phase Fission Heating and Separation of the Fissionable Material, Oak Ridge National Laboratory Memorandum CF 57-11-3, November 1, 1957. Classification AEC-C86, declassified December 21, 1959.

${ }^{2}$ Evans, R. D., The Atomic Nucleus, McGraw-Hill Book Co., Inc., 1955.

${ }^{3}$ Chapman, S., and Cowling. T. G., The Mathematical Theory of Non-Uniform Gases, Cambridge University Press, 1958.
} 